\title{
Bifurcation and branching of equilibria in reversible equivariant vector fields
}

\author{
Pietro-Luciano Buono \\ Faculty of Science, University of Ontario Institute of Technology \\ 2000 Simcoe St. North \\ Oshawa, ON L1H 7K4, Canada \\ Jeroen S.W. Lamb \\ Department of Mathematics, Imperial College London \\ London, SW7 2AZ, UK \\ Mark Roberts \\ Department of Mathematics \& Statistics, University of Surrey \\ Guildford, GU2 7XH, UK
}

January 7, 2008

\begin{abstract}
We study steady-state bifurcation in reversible equivariant vector fields. We assume an action on the phase space of a compact Lie group $G$ with a normal subgroup $H$ of index two, and study vector fields that are $H$-equivariant and have all elements of the complement $G \backslash H$ as time-reversal symmetries.

We focus on separable bifurcation problems that can be reduced to equivariant steady-state bifurcation problems, possibly with parameter symmetries. We describe both bifurcations of equilibria that arise when external parameters are varied, and branching of families of equilibria that may arise in the phase space when external parameters are fixed. We also show how our results apply to bifurcation problems for reversible relative equilibria and reversible (relative) periodic orbits.
\end{abstract}




\section{Introduction and summary}

Symmetry properties arise naturally and frequently in dynamical systems. In recent years, a lot of attention has been devoted to understanding and using the interplay between dynamics and symmetry properties. The type of symmetries that have been studied most thoroughly are those that do not involve a transformation of the independent variable (i.e. the 'time'-variable). Systems with such symmetries are usually called equivariant dynamical systems. Alternatively, there are also symmetry transformations that involve a transformation of the time-variable. In particular, time-reversibility (transformation of spatial variables and simultaneously an inversion $t \rightarrow-t$ of time) is a symmetry arising in many dynamical systems of interest. Examples are found in mechanical systems and in dynamical systems obtained from reductions of PDEs with symmetries involving a transformation of the independent variables. For a more extended discussion see for instance the survey by Lamb and Roberts [25].

In this paper we address the problem of steady-state bifurcation in dynamical systems that are equivariant and reversible. The corresponding problem of steady-state bifurcation in equivariant (non-reversible) systems has been studied extensively, see for instance Field [9] or Golubitsky et al. [19]. However the problem for reversible equivariant systems, has not been addressed systematically before, even though examples of steady-state bifurcations in reversible (equivariant) vector fields have been discussed by Politi et al. [32], Lamb and Capel [22], Teixeira [35], da Rocha Medrado and Teixeira [5], and Lim and McComb [28, 29]. The work of the Lim and McComb highlighted the existence of two types of steady-state bifurcations which they named null-separable and null-nonseparable bifurcations.

One of the problems in developing a systematic bifurcation theory for reversible equivariant systems has been the lack of suitable general framework. However the classification of linear reversible equivariant systems by Lamb and Roberts [26] opened the way for a systematic program of studies of reversible equivariant dynamical systems, extending the well established program for equivariant dynamical systems. In this paper we address the problem of steady-state bifurcation in reversible equivariant systems. For a treatment of Hopf bifurcation, see Vanderbauwhede [38] and Buzzi and Lamb [3].

Our main result shows that often (in the so called separable bifurcations) the reversibleequivariant steady-state bifurcation problem may be reduced to the setting of equivariant (nonreversible) steady-state bifurcation, possibly with parameter symmetries. More precisely, we find that in these cases a subset of the bifurcating branches are obtained from the analysis of such a steady-state bifurcation problem. In many cases of interest this subset provides the key to a complete description of the branching pattern. The remaining (nonseparable bifurcation) cases do not admit such a reduction, and must be studied using more general singularity theoretical tools.

As many applications of reversible equivariant dynamical systems can be found in Hamiltonian mechanics, one may view the theory developed here as an important step towards developing the corresponding theory in the reversible equivariant Hamiltonian setting. However, we want to emphasize that there are also examples of non-Hamiltonian reversible equivariant dynamical systems to which our theory applies, e.g. the Stokeslet fluid model $[17,30]$ and the rolling disk [21] (an example of a non-Hamiltonian mechanical system with nonholonomic constraints). 
In addition, dynamical systems obtained from reductions of PDEs with symmetries involving a transformation of the independent variables need not be Hamiltonian.

We now briefly introduce our general setting for reversible equivariant dynamical systems. We consider a vector field on a manifold $\mathcal{M}$ and a symmetry group $G$ acting on it. For $x \in \mathcal{M}$, the set

$$
G_{x}=\{g \in G \mid g \cdot x=x\}
$$

is a subgroup of $G$ called the isotropy subgroup of $x$. Assume that $G$ is a compact Lie group and $G$ is the isotropy subgroup of some point $x \in \mathcal{M}$. Then, Bochner's theorem [2] implies that, without loss of generality, a neighborhood of $x$ in $\mathcal{M}$ can be modelled by a vector space $V$ on which $G$ acts linearly and orthogonally.

Let $V$ be a vector space and let $f: V \times \mathbb{R}^{\ell} \rightarrow V$ be a smooth vector field. We consider the $\ell$-parameter family of dynamical systems

$$
\frac{d x}{d t}=f(x, \lambda),
$$

where $\lambda \in \mathbb{R}^{\ell}$ is an $\ell$-dimensional parameter vector.

Consider the following linear representations of $G$ :

$$
\begin{aligned}
\rho & : G \rightarrow \mathbf{O}(V) \\
\sigma & : G \rightarrow \mathbb{Z}_{2}=\{+1,-1\} \\
\rho_{\sigma} & : \quad G \rightarrow \mathbf{O}(V) ; \quad \rho_{\sigma}(g)=\sigma(g) \rho(g) .
\end{aligned}
$$

Note that $\sigma$ is a nontrivial $\mathbb{Z}_{2}$ representation.

Definition 1.1 The vector field (1) is $G$-reversible equivariant, or $(G, \sigma)$-equivariant, if for all $g \in G$

$$
f(\rho(g) x, \lambda)=\rho_{\sigma}(g) f(x, \lambda) .
$$

Note that our definition implies that $x(t)$ is a solution of (1) if and only if $\rho(g) x(\sigma(g) t)$ is a solution for all $g \in G$.

A $G$-reversible equivariant vector field is given by a $G$-reversible equivariant map $f: V \rightarrow V$ which commutes with two different, possibly non-isomorphic, representations of $G$ on its source and target. For some representations, a linear $G$-reversible equivariant map may be forced to have a nontrivial kernel. We call these forced kernels. The irreducible representations in the forced kernel determine the type of bifurcation problems that can appear - the so-called separable and nonseparable bifurcations mentioned above. The symmetry type of the forced kernel depends only on the representations.

In this paper, we work towards a steady-state bifurcation theory for reversible-equivariant dynamical systems: we aim to describe the set of equilibrium points

$$
\left\{x \in V, \lambda \in \mathbb{R}^{\ell} \mid f(x, \lambda)=0\right\} .
$$

near a given equilibrium solution $(0,0)$.

In addition to presenting a number of results regarding the structure of the bifurcation set, we also work through several examples illustrating our observations that have motivated the theory. 
Some of our results rely on tools from equivariant transversality theory, in particular the concepts of $G$-transversality.

The original definitions of $G$-transversality are found in Bierstone [1] and Field [6] in the context of smooth $G$-equivariant maps between arbitrary smooth $G$-manifolds. The equivalence between the definitions in the papers above is proved in Field [7]. Bierstone developed the theory in order to extend Mather's singularity theory to equivariant maps while Field's interest was in equivariant dynamics. In the context of equivariant bifurcation theory Field $[8,10,9]$ and Field and Richardson [12] have been successful in using $G$-transversality theory for the development of a general theory of equivariant bifurcation and to demonstrate that the Maximal Isotropy Subgroup Conjecture (MISC) is incorrect for finite reflection groups [12]. We use $G$-transversality theory as part of our reduction procedure to $G$-equivariant (non-reversible) bifurcation problems to obtain structurally stable zero sets (which we call organizing centres) of certain $G$-maps between non-isomorphic $G$-representations.

This paper is divided into two main parts. The first part outlines step-by-step our approach to reversible equivariant bifurcation problems, presenting our main results and illustrating them with examples.

We begin in Section 2 with a discussion of the sets of equilibria near nondegenerate equilibria. We then divide the study of reversible-equivariant bifurcation problems into two classes depending on the absence or presence of a forced kernel for the associated linear reversible-equivariant vector field. In the absence of a forced kernel we show in Section 3.1 that reversible-equivariant bifurcation problems are equivalent to equivariant ones.

In Section 3.2 we discuss bifurcations in the presence of a forced kernel, dividing them into two types that we call separable and non-separable (after Lim and McComb [28, 29]). In this paper we focus on separable bifurcations. Lemma 3.8 is our main result about separable steady-state bifurcations, showing that part of the solution set in these cases is identical (up to equivariant diffeomorphism) to the solution set of an associated equivariant bifurcation problem, possibly with parameter symmetries. Then, in Section 3.4 we discuss the important special case of a forced kernel on which $G$ acts trivially. The case of nontrivial action of $G$ on the forced kernel is discussed in Section 3.4 and Theorem 3.14 is a version of Lemma 3.8 in this context.

In Section 4 we discuss applications of our results to branching and bifurcation of relative equilibria and (relative) periodic obits.

The second part of the paper is more technical. In Section 5 we recall some properties of linear reversible equivariant vector fields, and discuss the correspondence between the occurrence of a forced kernel for such vector fields, and the existence of a linear invertible reversible equivariant map. In Section 6.1, we give a short introduction to $G$-transversality theory including some basic results on stratifications. Finally, in Section 6.2 a sufficient condition on the existence of organizing centres for any group action is given and other constructions of organizing centres are also presented, including examples.

\section{Nondegenerate local zero sets}

Let $\Sigma$ be an isotropy subgroup of $G$. It turns out that equilibria with isotropy subgroup $\Sigma$ may arise in families. To get a feel for this phenomenon we may view $f$ as a $G$-equivariant map from 
the vector space $V$, with $G$ acting as the representation $\rho$, to the vector space $V_{\sigma}$ which has the same dimension as $V$, but has $G$ acting as the representation $\rho_{\sigma}$ :

$$
f: V \times \mathbb{R}^{\ell} \rightarrow V_{\sigma}, \quad \text { with } \quad f \rho(g)=\rho_{\sigma}(g) f \quad \forall g \in G .
$$

A $G$-reversible equivariant map may thus be viewed as a $G$-equivariant map with different representations of $G$ on domain and target. Correspondingly, as an equivariant map, $f$ maps $\operatorname{Fix}_{V}(\Sigma)$ to $\operatorname{Fix}_{V_{\sigma}}(\Sigma)$, where as usual

$$
\operatorname{Fix}_{V}(\Sigma)=\{v \in V \mid \rho(g) v=v, \forall g \in \Sigma\}, \quad \operatorname{Fix}_{V_{\sigma}}(\Sigma)=\left\{v \in V_{\sigma} \mid \rho_{\sigma}(g) v=v, \forall g \in \Sigma\right\} .
$$

Note that if $\Sigma$ is an isotropy subgroup, then the set of points with isotropy subgroup $\Sigma$ in $\operatorname{Fix}_{V}(\Sigma)$ is open and dense. However, as $\rho$ may be nonisomorphic to $\rho_{\sigma}$, it may happen that $\operatorname{dim}_{\operatorname{Fix}_{V}}(\Sigma) \neq$ $\operatorname{dim} \operatorname{Fix}_{V_{\sigma}}(\Sigma)$, which is important when determining $f^{-1}(0)$. The following example illustrates this situation.

Example 2.1 $\left(\mathbb{Z}_{2}\right.$-reversible in $\left.\mathbb{R}^{3}\right)$ Let $V=\mathbb{R}^{3}$ and $G=\mathbb{Z}_{2}(R)$ act on $\mathbb{R}^{3}$ by $R .(x, y, z)=$ $(x, y,-z)$. We consider a $G$-reversible vector field in $V$, the general form of the germ at zero ${ }^{1}$ is given by:

$$
\dot{x}=p\left(x, y, z^{2}\right) z, \quad \dot{y}=q\left(x, y, z^{2}\right) z, \quad \dot{z}=r\left(x, y, z^{2}\right)
$$

where $p, q$ and $r$ are smooth functions.

The isotropy subgroup $\mathbb{Z}_{2}$ is such that $\operatorname{dim} \operatorname{Fix}_{V}\left(\mathbb{Z}_{2}\right)=2$ and $\operatorname{dim}_{\operatorname{Fix}} V_{V_{\sigma}}(\Sigma)=1$. Suppose that $\left(x_{0}, y_{0}, 0\right) \in \operatorname{Fix}\left(\mathbb{Z}_{2}\right)$ is an equilibrium; that is, $r\left(x_{0}, y_{0}, 0\right)=0$. Let

$$
f(x, y, z)=\left[\begin{array}{c}
p\left(x, y, z^{2}\right) z \\
q\left(x, y, z^{2}\right) z \\
r\left(x, y, z^{2}\right)
\end{array}\right]
$$

and define

$$
f_{\mathbb{Z}_{2}}=\left.f\right|_{\operatorname{Fix}_{V}\left(\mathbb{Z}_{2}\right)}: \operatorname{Fix}_{V}\left(\mathbb{Z}_{2}\right) \rightarrow \operatorname{Fix}_{V_{\sigma}}\left(\mathbb{Z}_{2}\right)
$$

In fact,

$$
f_{\mathbb{Z}_{2}}(x, y, 0)=\left[\begin{array}{c}
0 \\
0 \\
r(x, y, 0)
\end{array}\right]
$$

and if the nondegeneracy assumption $d r\left(x_{0}, y_{0}, 0\right) \neq 0$ is satisfied, then the implicit function theorem implies that $\left(x_{0}, y_{0}, 0\right)$ is part of a one-dimensional family of equilibria with the same isotropy subgroup. As the isotropy subgroup 1 does not contain a reversing symmetry, equilibria with isotropy subgroup 1 appear isolated. Therefore, equilibria with isotropy subgroup $\mathbb{Z}_{2}$ and 1 are generically bounded away from each other.

To keep track of this dimensional difference of fixed point sets, we introduce the notion of the $\sigma$-index of an isotropy subgroup $\Sigma \leq G$ :

\footnotetext{
${ }^{1}$ Since this paper discusses local bifurcations, the general form is always of the germ at zero. This is implicit throughout the rest of the paper
} 
Definition 2.2 (index) Let $\Sigma$ be a subgroup of $G$. Then, the $\sigma$-index of $\Sigma$ on $V$ is defined as

$$
s_{V}(\Sigma)=\operatorname{dim} \operatorname{Fix}_{V}(\Sigma)-\operatorname{dim}_{\operatorname{Fix}_{V_{\sigma}}}(\Sigma) .
$$

Note that this definition of index is also found in Field [11]. When it is clear from the context that we are dealing with indices on $V$ we write $s(\Sigma)$ instead of $s_{V}(\Sigma)$.

We further define $n_{\Sigma}$ as

$$
n_{\Sigma}=\operatorname{dim} \frac{N(\Sigma)}{\Sigma}
$$

where $N(\Sigma):=\{g \in G \mid g \Sigma=\Sigma g\}$ is the normalizer of $\Sigma$ in $G$.

Finally, if $\Sigma$ is the isotropy subgroup of a point $x$, we let $(\Sigma)$ denote the conjugacy class of $\Sigma$ in $G$, and say that $(\Sigma)$ is the isotropy type of $x$ (with respect to a representation $\rho$ of $G$ on $V$ ).

The following result gives the dimension of families of equilibria with a given isotropy subgroup in terms of the index.

Proposition 2.3 Let $f: V \times \mathbb{R}^{\ell} \rightarrow V$ be an $\ell$-parameter family of $G$-reversible equivariant vector fields and let

$$
d_{\Sigma}:=\ell+s(\Sigma)
$$

(1) If $d_{\Sigma} \geq n_{\Sigma}$ and $(x, 0)$ is an equilibrium with isotropy subgroup $\Sigma$, then equilibria with isotropy

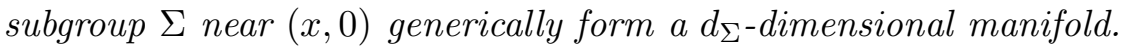

(2) If $d_{\Sigma}<n_{\Sigma}$, then generically $f$ has no equilibria with isotropy subgroup $\Sigma$.

Proof: $\quad$ Let $(x, 0) \in \operatorname{Fix}_{V}(\Sigma)$ be a point with isotropy subgroup $\Sigma$ such that $f(x, 0)=0$. Let $f_{\Sigma}$ be the the restriction of $f$ to $\operatorname{Fix}(\Sigma) \times \mathbb{R}^{\ell}$

$$
f_{\Sigma}: \operatorname{Fix}_{V}(\Sigma) \times \mathbb{R}^{\ell} \rightarrow \operatorname{Fix}_{V_{\sigma}}(\Sigma) .
$$

Note that the kernel of $\left(d f_{\Sigma}\right)_{(x, 0)}$ contains the tangent space at $(x, 0)$ to the $N(\Sigma) / \Sigma$ orbit through $(x, 0)$. However, if $d_{\Sigma} \geq n_{\Sigma}$ then generically $\left(d f_{\Sigma}\right)_{(x, 0)}$ is surjective and its kernel has dimension $d_{\Sigma}$. It follows that a neighbourhood of $(x, 0)$ in

$$
f_{\Sigma}^{-1}(0) \cap\left(\operatorname{Fix}_{V}(\Sigma) \times \mathbb{R}^{\ell}\right)
$$

is generically a $d_{\Sigma}$-dimensional manifold.

(ii) Since $d_{\Sigma}<n_{\Sigma}$, it follows that $\left(d f_{\Sigma}\right)$ cannot map $\operatorname{Fix}_{V}(\Sigma) \times \mathbb{R}^{\ell}$ surjectively to $\operatorname{Fix}_{V_{\sigma}}(\Sigma)$. Hence, generically $f_{\Sigma}^{-1}(0)$ is empty.

Proposition 2.3 only gives information about the dimension of the set of equilibria with constant isotropy subgroup $\Sigma$. Of particular interest is the generic set of equilibria to be found in a full open neighbourhood of a generic equilibrium point with isotropy $\operatorname{subgroup} \Sigma$ of a $G$-reversible equivariant vector field. We refer to this set as the nondegenerate local zero set of such an equilibrium. Equivariant transversality theory shows that the nondegenerate local zero set has the structure of a "stratified set". A stratification of a set is a locally finite partition of the set into smooth submanifolds called strata. In nondegenerate local zero sets each stratum has constant isotropy type. See Section 6.1 for a brief introduction to $G$-transversality and stratifications or 
for more details, see $[1,6,12,9,16]$. Knowledge of the structure of nondegenerate local zero sets is needed to obtain a complete picture of the bifurcation diagrams for $G$-reversible equivariant bifurcation problems.

In this paper we do not provide a general method to describe nondegenerate local zero sets. Instead we compute the set for specific examples when needed. Computing zero sets for $G$ equivariant maps between two arbitrary $G$-spaces requires the computation of minimal sets of homogeneous generators for maps, see for instance $[12,14]$ for examples with finite reflection groups or [40] for general computational and algorithmic aspects.

We illustrate the stratified structure of nondegenerate local zero sets below for some reversibleequivariant examples. Example 2.1 is a case where $\mathbb{Z}_{2}$ has positive index.

Example 2.4 ( $\mathbb{Z}_{2}$-reversible in $\mathbb{R}^{3} \times \mathbb{R}$ with negative index) Consider $G=\mathbb{Z}_{2}$ acting on $V=\mathbb{R}^{3}$ by $R .(x, y, z)=(x,-y,-z)$. We consider a $G$-reversible vector field in $V$. There are two isotropy subgroups $G$ and 1 . Their indices are $d_{G}=s(G)=-1$ and $d_{1}=s(1)=0$. Hence, when there is no external variable, equilibria with isotropy subgroup $G$ generically do not arise because of the negative index of $G$. Equilibria with isotropy subgroup 1 generically appear isolated.

In a one-parameter family, we have $\ell=1$ so that $d_{G}=s(G)+1=0$ and $d_{1}=s(1)+1=$ 1. Hence, in one-parameter families, equilibria with isotropy subgroup $G$ and 1 typically arise in zero and one-parameter families respectively. We can go a little further and show that in $\mathbb{R}^{3} \times \mathbb{R}$, equilibria with isotropy subgroup $G$ generically arise as a single point embedded in a one-dimensional family of equilibria with trivial isotropy subgroup.

A general $\mathbb{Z}_{2}$-reversible equivariant system $f$ has the form

$$
\dot{x}=p_{1}\left(x, y^{2}, z^{2}, \lambda\right) y+p_{2}\left(x, y^{2}, z^{2}, \lambda\right) z, \quad \dot{y}=q\left(x, y^{2}, z^{2}, \lambda\right), \quad \dot{z}=r\left(x, y^{2}, z^{2}, \lambda\right) .
$$

Suppose that $f(0,0,0,0)=0$. Generically, we can solve these equations locally using the implicit function theorem by $(x, y, \lambda)=\left(\phi_{1}\left(z^{2}\right), \phi_{2}\left(z^{2}\right) z, \phi_{3}\left(z^{2}\right)\right)$ with $\phi_{1}(0)=\phi_{3}(0)=0$. Hence, the equilibrium with isotropy subgroup $\mathbb{Z}_{2}$ is embedded in a one dimensional family of equilibria with trivial isotropy subgroup.

Example 2.5 $\left(\mathbf{S O}(2) \times \mathbb{Z}_{2}\right.$ in $\left.\mathbb{R}^{3} \times \mathbb{R}\right) \quad$ Consider $G=\mathbf{S O}(2) \times \mathbb{Z}_{2}(R)$ acting on $V=\mathbb{R}^{3} \simeq$ $\mathbb{C} \times \mathbb{R}$ by

$$
R .(z, x)=(z,-x) \quad \text { and } \quad \theta .(z)=\left(e^{i \theta} z, x\right), \quad \theta \in \mathbf{S O}(2) .
$$

We consider a $G$-reversible equivariant vector field on $V$, with $\sigma$-representation of $G$ :

$$
\sigma(R)=-1 \quad \text { and } \quad \sigma(\theta)=1 .
$$

The isotropy subgroups of $G$ are $G, \mathbf{S O}(2), \mathbb{Z}_{2}(R)$ and 1 . Here $n_{G}=n_{\mathbf{S O}(2)}=0$ and $n_{\mathbb{Z}_{2}}=n_{1}=1$. Furthermore, $d_{G}=s(G)=-1, d_{\mathbf{S O}(2)}=s(\mathbf{S O}(2))=0, d_{\mathbb{Z}_{2}(R)}=s\left(\mathbb{Z}_{2}(R)\right)=1$ and $d_{1}=s(1)=0$. Hence, since $d_{\mathbb{Z}_{2}(R)} \geq n_{\mathbb{Z}_{2}(R)}$, equilibria with isotropy subgroup $\mathbb{Z}_{2}(R)$ form a one-dimensional $\mathbf{S O}(2)$ group orbit of equilibria. Because $d_{\mathbf{S O}(2)}=n_{\mathbf{S O}(2)}$, equilibria with isotropy subgroup $\mathbf{S O}(2)$ are isolated. As $d_{G}<n_{G}$ and $d_{1}<n_{1}$, generically equilibria with isotropy subgroup $G$ and 1 do not appear. 
In a one-parameter family we have $\ell=1$ so that $d_{G}=0, d_{\mathbf{S O}(2)}=1, d_{\mathbb{Z}_{2}(R)}=2$ and $d_{1}=1$. As in the previous example, we study how these equilibria are interconnected. The general $\mathbf{S O}(2) \times \mathbb{Z}_{2}(R)$-reversible equivariant vector field $f$ is

$$
\dot{z}=p\left(x^{2},|z|^{2}, \lambda\right) x z, \quad \dot{x}=q\left(x^{2},|z|^{2}, \lambda\right) .
$$

If $f(0,0,0)=0$ then the origin $(0,0,0)$ is an equilibrium with isotropy subgroup equal to $G$. If $q_{\lambda}(0,0,0) \neq 0$ then it is embedded in a 1-dimensional family of $\mathbf{S O}(2)$ group orbits of equilibria with isotropy subgroups equal to $\mathbb{Z}_{2}(R)$. However, since $d_{\mathbf{S O}(2)}=1>0=n_{\mathbf{S O}(2)}$, it is also embedded in a one-dimensional family of equilibria with isotropy subgroup $\mathbf{S O}(2)$.

Since $d_{\Sigma}=n_{\Sigma}=1$, in a one-parameter family equilibria with isotropy subgroup 1 appear persistently as isolated $\mathbf{S O}(2)$-group orbits of dimension one.

Example 2.6 $\left(\mathbf{O}(2)\right.$ in $\left.\mathbb{R}^{2}\right) \quad$ Consider $G=\mathbf{O}(2)$ acting on $V=\mathbb{R}^{2} \simeq \mathbb{C}$ by

$$
R .(z)=\bar{z} \quad \text { and } \quad \theta .(z)=e^{i \theta} z, \quad \theta \in \mathbf{S O}(2) .
$$

We consider a $G$-reversible equivariant vector field on $V$, with $\sigma$-representation of $G$ :

$$
\sigma(R)=-1 \quad \text { and } \quad \sigma(\theta)=1 .
$$

The isotropy subgroups of $G$ are $G$ and $\mathbb{Z}_{2}(R)$. Here $n_{G}=0, n_{\mathbb{Z}_{2}}=0, d_{G}=s(G)=0$, and $d_{\mathbb{Z}_{2}(R)}=s\left(\mathbb{Z}_{2}(R)\right)=0$. Since $d_{\mathbb{Z}_{2}(R)}=n_{\mathbb{Z}_{2}(R)}$, equilibria with isotropy subgroup $\mathbb{Z}_{2}(R)$ are typically isolated. Such equilibria lie in $\mathbf{S O}(2)$ group orbits of equilibria all of which have different, but conjugate, isotropy subgroups. Moreover it can be shown that the $\mathbf{S O}(2)$-group orbit of equilibria is itself typically embedded in a one-parameter family of $R$-reversible relative equilibria passing transversally through $\operatorname{Fix} \mathbb{Z}_{2}$.

As $d_{G}=n_{G}=0$, equilibria with full isotropy subgroup $G$ generically arise as isolated points. In this example it is actually the limit point of a one-parameter family of $R$-reversible relative equilibria.

Example 2.7 $\left(\mathbb{T}_{d}\right.$-reversible equivariant in $\left.\mathbb{R}^{3}\right) \quad$ Let $G=\mathbb{T}_{d}$ be the full tetrahedral group acting on $V=\mathbb{R}^{3}$ by its standard representation as symmetries of the regular tetrahedron. The group can be decomposed $G=\mathbb{T}_{d}=\mathbb{T} \rtimes \mathbb{Z}_{2}(R)$ where $\mathbb{T}$ is the orientation preserving tetrahedral group generated by two generators $\gamma$ and $\kappa$, and $R$ is orientation reversing. The generators have the following representations in $V=\mathbb{R}^{3}$ :

$$
\rho(\gamma)=\left[\begin{array}{lll}
0 & 0 & 1 \\
1 & 0 & 0 \\
0 & 1 & 0
\end{array}\right], \quad \rho(\kappa)=\left[\begin{array}{ccc}
1 & 0 & 0 \\
0 & -1 & 0 \\
0 & 0 & -1
\end{array}\right] \quad \text { and } \quad \rho(R)=\left[\begin{array}{lll}
1 & 0 & 0 \\
0 & 0 & 1 \\
0 & 1 & 0
\end{array}\right] \text {. }
$$

The isotropy lattice of $\mathbb{T}_{d}$ is given in Figure 1. For each isotropy subgroup $\Sigma$ the index $s(\Sigma)$ is also indicated. Note that since $G$ is finite, we always have $n_{\Sigma}=0$. Now $\operatorname{dim} \operatorname{Fix}_{V_{\sigma}}(\Sigma)=\{0\}$ for $\Sigma=\mathbb{D}_{2}$ and $\Sigma=\mathbb{D}_{3}$. Hence, the equilibrium at the origin with isotropy subgroup $\mathbb{T}_{d}$ is embedded in one-dimensional families of equilibria with isotropy subgroups $\mathbb{D}_{2}$ and $\mathbb{D}_{3}$. Equilibria with isotropy subgroups $\mathbb{D}_{2}, \mathbb{D}_{3}, \mathbb{Z}_{2}(R)$ and $\mathbb{Z}_{2}(\kappa R)$ are typically locally embedded in one-dimensional manifolds of equilibria with the same isotropy subgroup. See Figure 2 for an illustration of the nondegenerate local zero set in $\mathbb{R}^{3}$. 


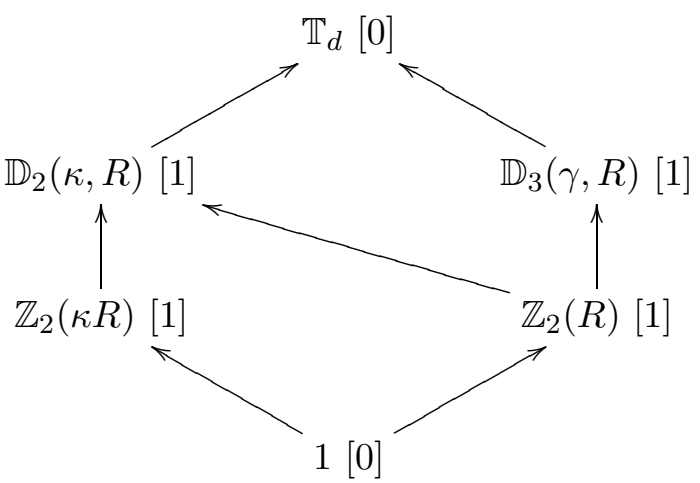

Figure 1: Isotropy lattice for the $\mathbb{T}_{d}$ action on $\mathbb{R}^{3}$ in Example 2.7. The indices of the isotropy subgroups are indicated in square brackets.

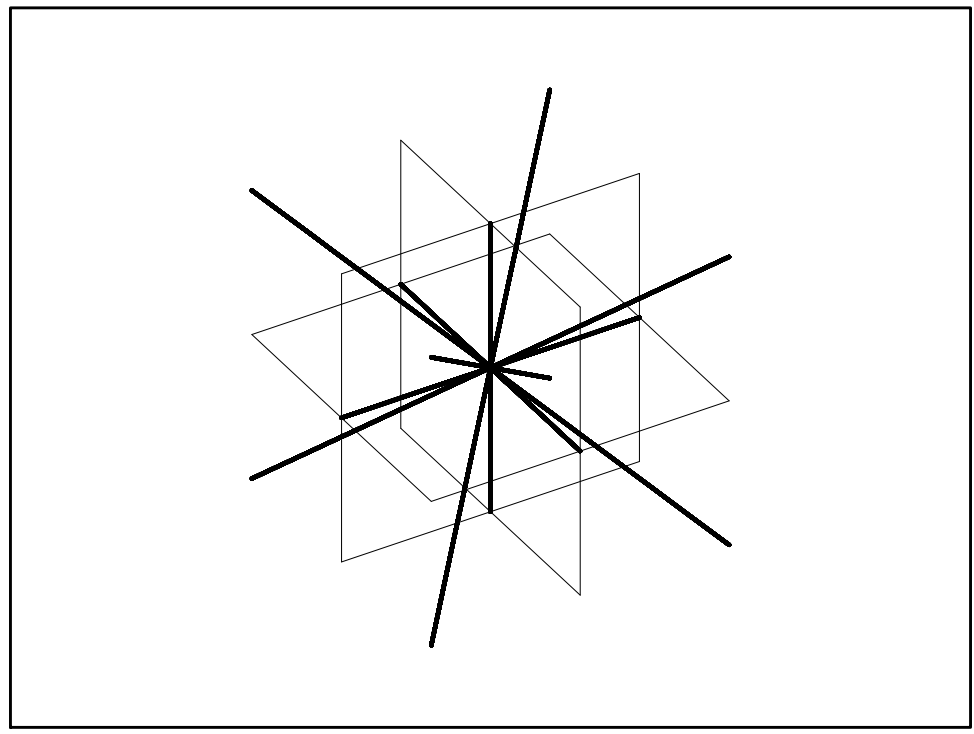

Figure 2: Sketch of the nondegenerate local zero set near 0 for the $\mathbb{T}_{d}$-reversible equivariant vector field in $\mathbb{R}^{3}$ discussed in Example 2.7. 


\section{Separable bifurcation and branching}

At a degenerate point of the set of equilibria, one of the two following situations may occur:

(i) The point is a local bifurcation point, i.e. the local zero set changes as we vary the external parameters;

(ii) the point is a branching point of the set of equilibria, i.e. the local zero set changes as we vary our reference point on the family of equilibria in phase space without varying external parameters.

Our aim is to describe bifurcation and branching of equilibria in families of $G$-reversible equivariant vector fields. In fact, bifurcation points are also branching points if we view $V \times \mathbb{R}^{\ell}$ as the phase space. Our discussion naturally deals with branching and bifurcation at the same time. However, to emphasize that we may have branching families of equilibria in phase space without varying external parameters, we tend to reserve the term "bifurcation" for cases where branching is induced by varying external parameters.

It is natural to address the question whether steady-state bifurcations of $G$-reversible equivariant vector fields are related to steady-state bifurcations of $G$-equivariant vector fields. We show that in certain circumstances, in the absence of a forced kernel, or when the representation of $G$ on the forced kernel is trivial, these problems are indeed very closely related. More generally our approach to separable bifurcations leads to steady state bifurcation problems for $G$-equivariant vector fields with parameter symmetries.

\subsection{Bifurcation in the absence of a forced kernel}

The following observation is fundamental to our approach to steady-state bifurcation and branching:

Lemma 3.1 Let $T: V \rightarrow V$ be a linear invertible $G$-reversible equivariant map. Then,

(i) $f: V \rightarrow V$ is $G$-reversible equivariant if and only if $\tilde{f}=T f$ is $G$-equivariant

(ii) $\tilde{f}(u, \lambda)=0$ if and only if $f(u, \lambda)=0$.

Proof: (i) If $f$ is $G$-reversible equivariant, then

$$
\tilde{f}(g x)=T \sigma(g) g f(x)=\sigma(g) T g f(x)=\sigma(g)^{2} g T f(x)=g \tilde{f}(x) .
$$

A similar calculation shows the converse. (ii) Follows from invertibility of $T$.

Hence reversible equivariant and equivariant steady-state bifurcation problems are equivalent whenever there exists a linear reversible equivariant invertible map $T$. This is equivalent to the representation of $G$ being such that a linear $G$-reversible equivariant map $L$ has no forced kernel.

Let $L: V \rightarrow V$ be a linear $G$-reversible equivariant map commuting with the representations $\rho$ and $\rho_{\sigma}$ of $G$ on $V$. If $\rho$ and $\rho_{\sigma}$ are nonisomorphic irreducible representations then Schur's lemma 
implies $L \equiv 0$ and $L$ is forced to have a kernel by the representations. For general representations $\rho$ and $\rho_{\sigma}$, the map $L$ has a nontrivial kernel forced by the group representations if the isotypic decompositions of the representations $\rho$ and $\rho_{\sigma}$ are not isomorphic. The forced kernel for a linear reversible equivariant map $L$ is the isomorphism class (as a group representation) of the lowest dimensional kernel that a reversible equivariant map can have between these representations. A more technical definition and further details are given in Section 5.

The existence of forced kernels for linear reversible equivariant maps depends only on the representation of $G$ on $V$. This observation, in combination with Lemma 3.1, leads to our first theorem:

Theorem 3.2 (Bifurcation in absence of forced kernel) Let $f: V \times \mathbb{R}^{\ell} \rightarrow V$ be an $\ell$ parameter family of $G$-reversible equivariant vector fields, with $f(0,0)=0$. Then, if the representation of $G$ on $V$ is such that $d_{V} f(0,0)$ has no forced kernel, the steady-state bifurcations from $(0,0)$ are equivalent to steady-state bifurcations of an $\ell$-parameter family of $G$-equivariant vector fields. In particular, the set of equilibria in $V \times \mathbb{R}^{\ell}$ near a $G$-reversible equivariant steady-state bifurcation point is locally diffeomorphic to the set of equilibria in $V \times \mathbb{R}^{\ell}$ near a corresponding G-equivariant steady-state bifurcation point.

We illustrate the theorem with an elementary example.

\section{Example $3.3\left(\mathbb{Z}_{2}\right.$-reversible steady-state bifurcation: no forced kernel)}

Let $f$ be a $\mathbb{Z}_{2}$-reversible vector field in $\mathbb{R}^{2 m}$, where $\mathbb{Z}_{2}$ is generated by $R: \mathbb{R}^{m} \times \mathbb{R}^{m} \rightarrow \mathbb{R}^{m} \times \mathbb{R}^{m}$, with $R(x, y)=(x,-y)$. There exists a $\mathbb{Z}_{2}$-reversible linear invertible map $T(x, y)=(y, x)$. Hence, generically, steady-state bifurcations of $F$ are in one-to-one correspondence with steady-state bifurcations of a $\mathbb{Z}_{2}$-equivariant vector field $g$ in $\mathbb{R}^{2 m}$. In one-parameter families, such bifurcations are generically symmetry preserving folds (saddle-nodes or saddle-centres), or symmetry breaking pitchforks.

Example 3.4 (Self-dual representations) An irreducible representation $\rho: G \rightarrow \mathbf{O}(V)$ is self-dual if $V$ and $V_{\sigma}$ are isomorphic representations, see Lamb and Roberts [26] for more details. A consequence of Schur's lemma is that for any self-dual irreducible representation $V$ there exists an invertible reversible-equivariant linear map $T: V \rightarrow V_{\sigma}$. Therefore, if $f: V \rightarrow V$ is a $G$ reversible equivariant bifurcation problem where the isotypic decomposition of $V$ contains only self-dual irreducible representations, then there exists an invertible reversible-equivariant linear map $T: V \rightarrow V$ and Theorem 3.2 applies.

\subsection{Separability and organizing centres in the presence of a forced kernel}

The existence of forced kernels is closely related to the fact that in reversible equivariant vector fields equilibria may arise in families. Theorem 3.2 describes a reduction to standard equivariant steady-state bifurcation theory in the case of no forced kernel. It remains to consider the problem when there is a forced kernel.

In this paper we only consider bifurcations in which we can 'separate' the forced kernel from its complement in the full kernel of $d_{V} f$ at the bifurcation point. We begin by defining what we mean by this. 
Assume that $(0,0)$ is an equilibrium point of the vector field $f(x, \lambda)$. Decompose $V$ into the direct sum of two representations:

$$
V=W_{1} \oplus W_{2},
$$

where $W_{2}$ is isomorphic to the forced kernel and $W_{1}$ is a complement. Note that $W_{1}$ has the property that there exists an invertible linear reversible equivariant map $T: W_{1} \rightarrow W_{1}$.

Definition 3.5 We say that $(0,0)$ is a separable bifurcation point if ker $d_{V} f(0,0) \cong W_{2} \oplus U$ where $U$ is a representation of $G$ such that:

(1) $\operatorname{dim} U>0$, and

(2) The decompositions of $U$ and $W_{2}$ into irreducible representations of $G$ have no isomorphic components.

This generalises the definition of separable bifurcations introduced by Lim and McComb [28, 29]. Note that a bifurcation with no forced kernel, as described above, is trivially separable.

In Proposition 5.4 we prove that for separable bifurcations we can always make a $\lambda$-dependent $G$-equivariant linear change of coordinates on $V$ so that ker $d_{V} f(0,0)$ contains $W_{2}$ :

$$
d_{W_{2}} f(0, \lambda)=0 \text { for all } \lambda .
$$

Henceforth we always assume this.

We now decompose the equation $f\left(w_{1}, w_{2}, \lambda\right)=0$ into two parts

$$
\left\{\begin{array}{l}
f_{1}\left(w_{1}, w_{2}, \lambda\right)=0 \\
f_{2}\left(w_{1}, w_{2}, \lambda\right)=0
\end{array}\right.
$$

where $f_{1}: W_{1} \oplus W_{2} \times \mathbb{R}^{\ell} \rightarrow W_{1}$ and $f_{2}: W_{1} \oplus W_{2} \times \mathbb{R}^{\ell} \rightarrow W_{2}$. By using an invertible linear reversible equivariant map $T: W_{1} \rightarrow W_{1}$ these equations can be replaced by the equivalent set:

$$
\left\{\begin{array}{l}
\tilde{f}_{1}\left(w_{1}, w_{2}, \lambda\right)=0 \\
f_{2}\left(w_{1}, w_{2}, \lambda\right)=0
\end{array}\right.
$$

where

$$
\tilde{f}_{1}\left(w_{1}, w_{2}, \lambda\right):=T f_{1}\left(w_{1}, w_{2}, \lambda\right)
$$

is $G$-equivariant:

$$
\tilde{f}_{1}\left(\rho_{1}(g) w_{1}, \rho_{2}(g) w_{2}, \lambda\right)=\rho_{1}(g) \tilde{f}_{1}\left(w_{1}, w_{2}, \lambda\right), \quad \forall g \in G
$$

with $\rho_{i}:=\left.\rho\right|_{W_{i}}$. At the same time recall that for all $g \in G$,

$$
f_{2}\left(\rho_{1}(g) w_{1}, \rho_{2}(g) w_{2}, \lambda\right)=\rho_{\sigma, 2}(g) f_{2}\left(w_{1}, w_{2}, \lambda\right)
$$

where $\rho_{\sigma, 2}$ is the restriction of $\rho_{\sigma}$ to $W_{2}$.

Consider a point $\left(w_{1}, w_{2}, \lambda\right)$ in $f_{2}^{-1}(0)$ with isotropy subgroup $G$. If $\ell$ is sufficiently large then locally such points typically occur in $\left(d_{G}+\operatorname{dim} \operatorname{Fix}_{W_{1}} G\right)$-dimensional manifolds. 
The local structure of the whole of $f_{2}^{-1}(0)$ may depend on the point chosen in this set. Our aim is to describe a $G$-invariant subset of $f_{2}^{-1}(0)$ which after substitution into the $\tilde{f}_{1}=0$ equation reduces the separable bifurcation problem $f=0$ to a $G$-equivariant bifurcation problem on $W_{1}$, possibly with parameter symmetries. In this way we obtain an equivalence between a subset of the solution set of $f=0$ and the full solution set of a $G$-equivariant bifurcation problem. Let $\pi_{1}: W_{1} \times W_{2} \times \mathbb{R}^{\ell} \rightarrow W_{1}$ be the projection to the component $W_{1}$, we now come to the definition of organizing centre.

Definition 3.6 (Organizing centre) A point $(x, 0) \in V \times \mathbb{R}^{\ell}$ with isotropy subgroup $G$, is an organizing centre for a G-reversible-equivariant steady-state bifurcation problem $f=0$ if near $(x, 0)$ the set $f_{2}^{-1}(0)$ contains a smooth $G$-invariant submanifold $K$ of $V \times \mathbb{R}^{\ell}$ with the following property: the restriction of $\pi_{1}$ to $K$ is a submersion at $(x, 0)$.

Remark 3.7 Notice that there is no mention in Definition 3.6 of the persistence of the manifold $K$ to small perturbations in $f_{2}$. This has been done on purpose as to keep the technical details to a minimum in this presentation. In Section 6.2 we show how to construct persistent manifolds $K$ using $G$-transversality theory. All the organizing centres in our examples are obtained using this method.

Theorem 6.4 shows that bifurcation problems with organizing centers can be found for any pair $(V, \sigma)$ provided the initial parameter space $\mathbb{R}^{\ell}$ is large enough. The proof is an application of the implicit function theorem and thus the manifold $K$ is persistent to small perturbations.

Recall that a differentiable map $f$ from an $m$-manifold $M$ to an $n$-manifold $N$ is called a submersion at a point $p$ of $M$ if its differential $d f(p)$ is surjective, or equivalently if rank $d f(p)=$ $\operatorname{dim} N$. Note that the map $\pi_{1}: K \rightarrow W_{1}$ is $G$-equivariant and so ker $d \pi_{1}(x, 0) \cong T_{(x, 0)} K \cap\left(W_{2} \times \mathbb{R}^{\ell}\right)$ is $G$-invariant and isomorphic to a subrepresentation of $W_{2} \times \mathbb{R}^{\ell}$.

Lemma 3.8 If $(x, 0)$ is an organizing centre, and $K \subset V \times \mathbb{R}^{\ell}$ a submanifold as described in Definition 3.6, then the restriction of the equation $\tilde{f}_{1}=0$ to $K$ is $G$-diffeomorphic to an equivariant bifurcation problem $\tilde{f}_{\text {red }}: W_{1} \times \operatorname{ker} d \pi_{1}(x, 0) \rightarrow W_{1}$.

Proof: Let $u$ be the coordinate on ker $d \pi_{1}(x, 0)$. Then, an application of the implicit function theorem to $\pi_{1}$ guarantees that we can write

$$
K=\left\{\left(w_{1}, w_{2}, \lambda\right) \mid\left(w_{2}, \lambda\right)=\phi\left(w_{1}, u\right) \text { where } \phi \text { is smooth and } G \text {-equivariant }\right\} .
$$

locally near $(x, 0)$. We define the bifurcation problem $f_{\text {red }}$ near $(x, 0)$ as

$$
f_{\text {red }}\left(w_{1}, u\right)=\tilde{f}_{1}\left(w_{1}, \phi\left(w_{1}, u\right)\right) .
$$

Let $\tilde{B}$ be the zero set of $\tilde{f}_{1}$ restricted to $K$ and $B^{\prime}$ be the zero set of $f_{\text {red }}$. The mapping

$$
\begin{aligned}
\Psi: \quad & B^{\prime} \rightarrow \tilde{B} \\
& \left(w_{1}, u\right) \rightarrow\left(w_{1}, w_{2}, \lambda\right)=\left(w_{1}, \phi\left(w_{1}, u\right)\right)
\end{aligned}
$$

is a local $G$-diffeomorphism near $(x, 0)$ since it is smooth and with smooth inverse given by the projection from $K$ to the domain of $\phi$ near $(x, 0)$. The $G$-equivariance of $\Psi$ is a consequence of the $G$-equivariance of $\phi$. 
In general $G$ acts non-trivially on the parameter space ker $d \pi_{1}(x, 0)$ and so $\tilde{f}_{\text {red }}$ is a bifurcation problem with parameter symmetries. If, as in many of our examples (i.e. Example 3.11), $\operatorname{dim} K=$ $\operatorname{dim} W_{1}+\ell$ then $\operatorname{dim} \operatorname{ker} d \pi_{1}(x, 0)=\ell$ and $\operatorname{ker} d \pi_{1}(x, 0)$ can itself be identified with $\mathbb{R}^{\ell}$. This is equivalent to solving the equation $f_{2}\left(w_{1}, w_{2}, \lambda\right)=0$ for $w_{2}$ as a function of $w_{1}$ and $\lambda$.

\subsection{Bifurcation with trivial forced kernel symmetry}

We first apply the strategy described in the previous subsection to the situation when $W_{2}$ is a trivial representation, in which case we speak of the bifurcation problem having trivial forced kernel symmetry. Under this assumption the representation of $G$ on $\operatorname{ker} d \pi_{1}(x, 0)$ is also trivial and so $\tilde{f}_{\text {red }}=0$ is a $G$-equivariant steady-state bifurcation problem on $W_{1}$ with trivial action on the parameter space.

The equation $\tilde{f}_{1}\left(w_{1}, w_{2}, \lambda\right)=0$ can be regarded as an equivariant bifurcation problem on $W_{1}$ parametrised by $W_{2} \times \mathbb{R}^{\ell}$ and so its bifurcating branches generically come in $\operatorname{dim} W_{2}+\ell$ dimensional families. The bifurcation diagram for the full reversible equivariant problem is obtained by intersecting these branches with $f_{2}^{-1}(0)$.

We split the problem of determining what these intersections look like into two cases, depending on whether or not the isotropy subgroup $\Sigma$ contains a reversing symmetry.

First let $\Sigma$ be an isotropy subgroup of $G$ which contains a reversing symmetry. Then since $\rho_{2}(g)$ acts as $\sigma(g) I$ on $W_{2, \sigma}$, from (11) it follows that $f_{2}\left(w_{1}, w_{2}, \lambda\right)=0$ for all $w_{1} \in \operatorname{Fix}_{W_{1}}(\Sigma)$ and $w_{2} \in W_{2}$, and so

$$
f_{2}^{-1}(0) \cap \operatorname{Fix}_{V}(\Sigma)=\operatorname{Fix}_{V}(\Sigma) \oplus \mathbb{R}^{\ell}=\operatorname{Fix}_{W_{1}}(\Sigma) \oplus W_{2} \oplus \mathbb{R}^{\ell} .
$$

It follows that the part of the bifurcation diagram of $\tilde{f}_{1}=0$ consisting of equilibria with isotropy subgroups containing reversing symmetries is identical to the equivalent part of the bifurcation diagram for the full reversible equivariant problem $f=0$. The branches typically have dimension equal to $\operatorname{dim} W_{2}+\ell$.

In case $\Sigma$ does not contain any reversing symmetries we assume that the bifurcation point is an organizing centre and that $K \subset V \times \mathbb{R}^{\ell}$ is a submanifold with the properties described in Definition 3.6. Since $G$ acts trivially on ker $d \pi_{1}(x, 0)$, by Lemma 3.8 the restriction of $\tilde{f}_{1}=0$ to $K$ is equivalent to an equivariant bifurcation problem $\tilde{f}_{\text {red }}\left(w_{1}, \nu\right)=0$ on $W_{1} \times \mathbb{R}^{k}$ where $k=\operatorname{dim} K-\operatorname{dim} W_{1}$. This typically has bifurcating branches of dimension $k$ and correspond to bifurcating branches of the full reversible equivariant bifurcation problem $f=0$ of the same dimension $k$.

In the case of an isotropy subgroup that contains a reversing symmetry, the $k$-dimensional branch coming from $K$ is embedded in the larger $\operatorname{dim} W_{2}+\ell$-dimensional branch described above. At least part of the bifurcation diagram for the reversible equivariant problem $f=0$ looks like that for the equivariant problem $\tilde{f}_{\text {red }}=0$, but with the branches with isotropy subgroups containing reversing symmetry 'fattened-up' to have dimension $\operatorname{dim} W_{2}+\ell$. This following result formalises this.

Theorem 3.9 (Separable bifurcation with trivial forced kernel symmetry) Let $f: V \times$ $\mathbb{R}^{\ell} \rightarrow V$ be an $\ell$-parameter family of $G$-reversible equivariant vector fields, with $f(0,0)=0$. 
Suppose that $f$ has a separable bifurcation at $(0,0)$ and that the representation of $G$ on the forced kernel of $d_{V} f(0,0)$ is trivial. Let $B$ denote the set of equilibria in a small neighbourhood of $(0,0)$. Write $B=B_{0} \cup B_{r}$, where $B_{r}$ contains all equilibria whose isotropy subgroup contains a reversing symmetry and $B_{0}$ is the remainder. Then:

(1) The subset of $B_{r}$ consisting of equilibria with isotropy subgroup $\Sigma$ is generically a smooth submanifold of $V$ of dimension equal to $\operatorname{dim} W_{2}+\ell$.

(2) If $(0,0)$ is an organising centre then there exists a smooth $G$-invariant submanifold $K \subset$ $V \times \mathbb{R}^{\ell}$ such that $B_{0}=B \cap K$ is equivariantly diffeomorphic to the bifurcation diagram of a $G$-equivariant bifurcation problem $\tilde{f}_{\text {red }}: W_{1} \times \mathbb{R}^{k} \rightarrow W_{1}$ with

$$
0 \leq k=\operatorname{dim} K-\operatorname{dim} W_{1} \leq \operatorname{dim} W_{2}+\ell .
$$

Typically the branches of equilibria in $K$ have dimension $k$.

If we are not at an organizing centre, but near it, one observes only the branches whose isotropy subgroups contain a reversing symmetry. In fact, branches can be found in phase space, without the need to vary parameters.

Corollary 3.10 (Branching of equilibria in phase space) Consider the context of Theorem 3.9, with $\ell=0$. Let $k$ be positive such that $k \leq \operatorname{dim} W_{2}$. Then a $G$-invariant equilibrium of $f$ is locally contained in a $\left(\operatorname{dim} W_{2}\right)$-dimensional set of branching equilibria that is $G$-diffeomorphic to the set

$$
B \simeq B_{r}^{\prime} \times \mathbb{R}^{\operatorname{dim} W_{2}-k}
$$

where $B_{r}^{\prime}$ is $G$-diffeomorphic to the subset of a k-parameter family of $G$-equivariant vector fields on $W_{1}$, composed of the union of branches whose isotropy subgroups contain a reversing symmetry.

We refer to this phenomenon as branching of equilibria in phase space.

To illustrate the result of Theorem 3.9 and Theorem 3.10 we discuss some elementary examples.

Example $3.11\left(\mathbb{Z}_{2}\right.$-symmetry breaking pitchfork in $\left.\mathbb{R}^{3} \times \mathbb{R}\right)$ As in Example 2.1 we consider $\mathbb{Z}_{2}$-reversible vector fields on $\mathbb{R}^{3}$, where $\mathbb{Z}_{2}$ is generated by $R .(x, y, z)=(x, y,-z)$. For these we can fix

$$
W_{1}=\{(x, y, z) \mid x=0\} \quad W_{2}=\{(x, y, z) \mid y=z=0\} .
$$

The space $W_{2}$ is the forced kernel, with the trivial action of $\mathbb{Z}_{2}$. Adapting the notation of Example 2.1 , the assumption that the forced kernel is always equal to $W_{2}$ implies that

$$
r_{x}(0,0,0, \lambda)=0
$$

for all values of any parameter $\lambda$. This assumption can be made without loss of generality. The origin $(x, y, z, \lambda)=(0,0,0,0)$ is a separable bifurcation point if in addition

$$
p(0,0,0,0)=q(0,0,0,0)=0 .
$$




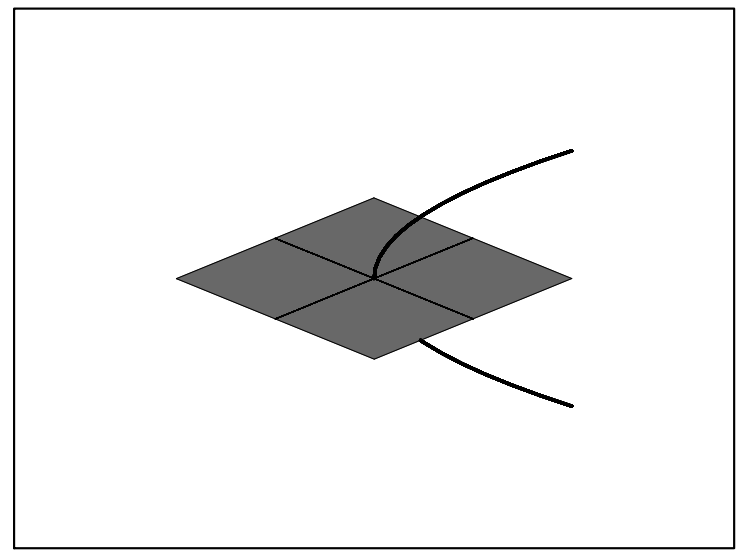

Figure 3: Projection of $\mathbb{Z}_{2}$-symmetry breaking pitchfork bifurcation in $\mathbb{R}^{3} \times \mathbb{R}$ to $\mathbb{R}^{2} \times \mathbb{R}$. The equivariant $\mathbb{Z}_{2}$-symmetry breaking pitchfork bifurcation diagram forms the basis of this set. The $\mathbb{Z}_{2}$-invariant branch has dimension two and the branch with trivial isotropy subgroup has dimension one.

Then,

$$
f_{2}(x, y, z, \lambda)=p\left(x, y, z^{2}, \lambda\right) z .
$$

Generically we may assume that, say, $p_{x}(0,0,0,0) \neq 0$ and so, by the implicit function theorem, we can solve $f_{2}=0$ for $x$ as a function of $y, z^{2}$ and $\lambda$ in a neighbourhood of the origin. This defines the submanifold $K$ in the definition of the organizing centre. Note that $\operatorname{dim} K=\operatorname{dim} W_{1}+\ell$, and so $k=\ell$ in Theorem 3.9. Substituting this into

$$
f_{1}(x, y, z, \lambda)=\left(q\left(x, y, z^{2}, \lambda\right) z, r\left(x, y, z^{2}, \lambda\right)\right)
$$

and composing with the reversible equivariant linear map $T(y, z)=(z, y)$ yields a reduced $\mathbb{Z}_{2^{-}}$ equivariant bifurcation problem on $W_{1}$ :

$$
\tilde{f}_{\text {red }}(y, z, \lambda)=\left(r\left(x\left(y, z^{2}, \lambda\right), y, z^{2}, \lambda\right), q\left(x\left(y, z^{2}, \lambda\right), y, z^{2}, \lambda\right) z\right) .
$$

Theorem 3.9 states that $B_{0}$ is diffeomorphic to the branching solutions of the generic $\mathbb{Z}_{2^{-}}$ symmetry breaking bifurcation diagram given by $\tilde{f}_{\text {red }}=0$. Thus, the bifurcation set $B$ in $\mathbb{R}^{3} \times$ $\mathbb{R}$ consists of a set diffeomorphic to the pitchfork bifurcation diagram described above, with additional one-dimensional "fattening" of the branch with isotropy subgroup $\mathbb{Z}_{2}$. The set is illustrated in Figure 3.

Example $3.12\left(\mathbb{D}_{2}\right.$-symmetry breaking pitchfork branching in $\left.\mathbb{R}^{3}\right)$ Consider a $\mathbb{D}_{2}$ reversible equivariant vector field in $\mathbb{R}^{3}$, generated by the reversing symmetries

$$
R_{1} \cdot(x, y, z)=(x, y,-z) \text { and } R_{2} \cdot(x, y, z)=(x,-y, z)
$$

with $\sigma\left(R_{1}\right)=\sigma\left(R_{2}\right)=-1$. A simple computation shows that a generic linear map commuting with the action of $\mathbb{D}_{2}$ on domain and range has a one-dimensional forced kernel. We can choose $W_{1}=\{(x, y, z) \mid x=0\}$ and $W_{2}=W_{1}^{\perp}$. 

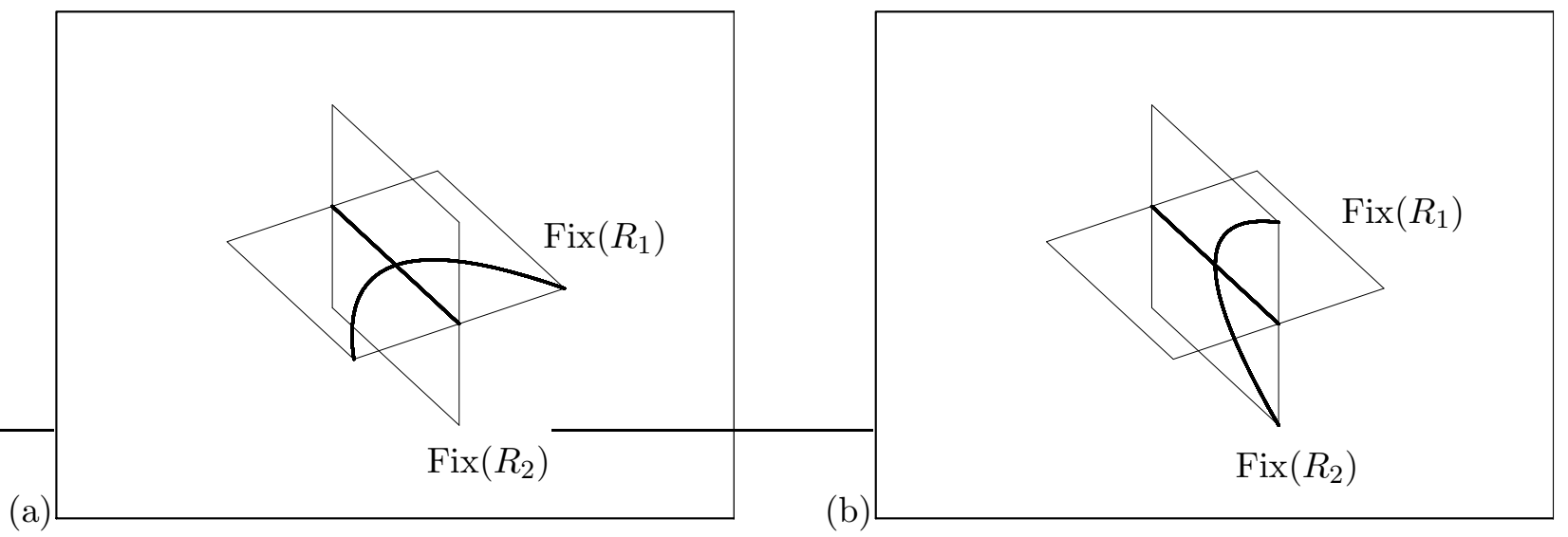

Figure 4: Generic $\mathbb{D}_{2}$-symmetry breaking pitchfork branching in $\mathbb{R}^{3}$, as discussed in Example 3.12. The figures illustrate generic pitchfork branching from $\mathbb{D}_{2}$ to (a) $\mathbb{Z}_{2}\left(R_{1}\right)$, and (b) $\mathbb{Z}_{2}\left(R_{2}\right)$.

We have $s\left(\mathbb{D}_{2}\right)=s\left(\mathbb{Z}_{2}\left(R_{1}\right)\right)=s\left(\mathbb{Z}_{2}\left(R_{2}\right)\right)=1$. Hence, generically equilibria with isotropy subgroup $\mathbb{D}_{2}, \mathbb{Z}_{2}\left(R_{1}\right)$ and $\mathbb{Z}_{2}\left(R_{2}\right)$ arise in one-dimensional families.

Now, we consider a one-parameter family of such vector fields, and separable bifurcation from an equilibrium point with isotropy subgroup $\mathbb{D}_{2}$. The reduced $\mathbb{D}_{2}$-equivariant generic steady-state bifurcation has a pitchfork bifurcation diagram with a bifurcating branch of solutions with isotropy subgroup $\mathbb{Z}_{2}\left(R_{1}\right)$ or $\mathbb{Z}_{2}\left(R_{2}\right)$. In $\mathbb{R}^{3} \times \mathbb{R}$, the bifurcation set of equilibria is a homogeneously fattened version of the pitchfork bifurcation diagram, as the isotropy subgroups of all branches contain a reversing symmetry, yielding two-dimensional branches with a one-dimensional intersection.

We illustrate Theorem 3.10 about branching in phase space by fixing a value of the parameter. Then, we may observe a persistent pitchfork branching of equilibria, breaking from isotropy subgroup $\mathbb{D}_{2}$ to $\mathbb{Z}_{2}\left(R_{1}\right)$ or $\mathbb{Z}_{2}\left(R_{2}\right)$. In Theorem 3.10 , we have $k=1$ since the pitchfork is codimension 1 and the set $B \simeq B_{r}^{\prime} \times \mathbb{R}^{0}$ where $B_{r}^{\prime}$ is $G$-diffeomorphic to the pitchfork bifurcation. These branching sets of equilibria are illustrated in Figure 4.

Example $3.13\left(\mathbb{D}_{4}\right.$ branching and bifurcation in $\left.\mathbb{R}^{3} \times \mathbb{R}\right) \quad$ Consider a $\mathbb{D}_{4}$-reversible equivariant vector field in $\mathbb{R}^{3}$, generated by the reversing symmetries

$$
\kappa_{1} \cdot(x, y, z)=(x,-y, z) \text { and } \kappa_{2} \cdot(x, y, z)=(y, x, z)
$$

with $\gamma=\kappa_{1} \kappa_{2}$ and

$$
\sigma\left(\kappa_{1}\right)=-\sigma\left(\kappa_{2}\right)=-1 .
$$

We set $W_{1}=\{(x, y, z) \mid z=0\}$ since this subrepresentation is self-dual, therefore $W_{2}=\{(x, y, z) \mid$ $x=y=0\}$ is the forced kernel.

The lattice of isotropy subgroups along with indices are given in Figure 6. Hence, generically equilibria with isotropy subgroups $\mathbb{D}_{4}, \mathbb{D}_{2}\left(\kappa_{1}, \gamma^{2}\right), \mathbb{Z}_{2}\left(\kappa_{1}\right)$ arise in one-dimensional families, other equilibria typically arise isolated. In a one-parameter family of such vector fields, we consider a separable bifurcation from an equilibrium point with isotropy subgroup $\mathbb{D}_{4}$. The reduced $\mathbb{D}_{4}$-equivariant generic steady-state bifurcation in $\mathbb{R}^{2} \times \mathbb{R}$ has a bifurcation set with bifurcating 

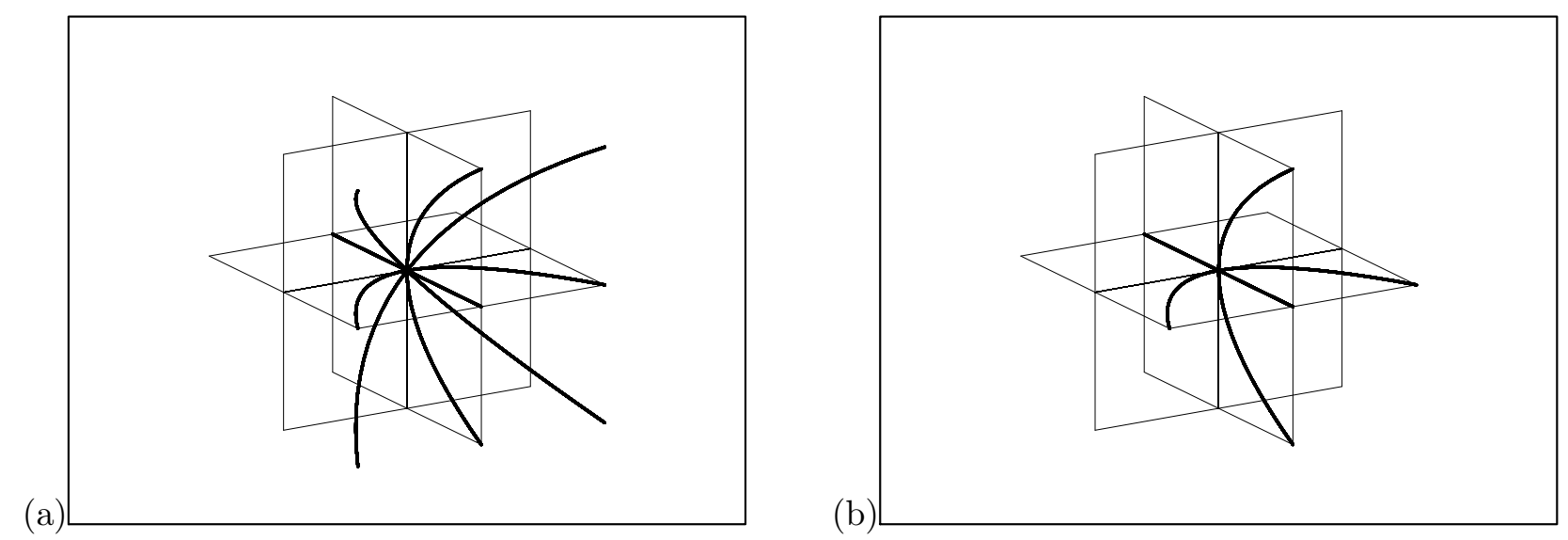

Figure 5: Steady-state bifurcation and branching for a $\mathbb{D}_{4}$-reversible equivariant vector field in $\mathbb{R}^{3} \times \mathbb{R}$, as discussed in Example 3.13. (a) Bifurcation set for the reduced bifurcation problem in $\mathbb{R}^{2} \times \mathbb{R}(\mathrm{b})$ Branching of equilibria in the phase space $\mathbb{R}^{3}$ at a fixed value of the parameter.

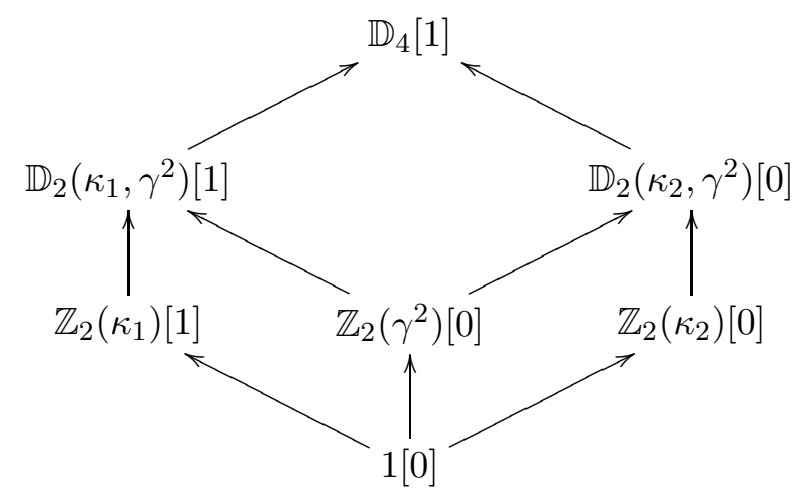

Figure 6: Isotropy lattice for the $\mathbb{D}_{4}$ action on $\mathbb{R}^{3}$ with isotropy subgroups indices

branches of solutions with isotropy subgroup $\mathbb{Z}_{2}\left(\kappa_{1}\right)$ and $\mathbb{Z}_{2}\left(\kappa_{2}\right)$ (and their conjugate solutions $\mathbb{Z}_{2}\left(\kappa_{2} \gamma\right)$ and $\left.\mathbb{Z}_{2}\left(\gamma \kappa_{1}\right)\right)$, see Figure $5(\mathrm{a})$. For the full equation, in $\mathbb{R}^{3} \times \mathbb{R}$, the bifurcation set of equilibria is a fattened version of the bifurcation set for the reduced problem, where all branches whose isotropy subgroup contains a reversing symmetry are two-dimensional (in $\mathbb{R}^{3} \times \mathbb{R}$ ), whereas the remaining branches are one-dimensional. Consequently (following Theorem 3.10), at a fixed value of the parameter we may observe a persistent branching point, at which in the phase space $\mathbb{R}^{3}$ a one-dimensional branch of equilibria with isotropy subgroup $\mathbb{D}_{4}$ intersects a one-dimensional family of equilibria with isotropy subgroup $\mathbb{Z}_{2}\left(\kappa_{1}\right)$ (and a one-dimensional family with conjugate isotropy subgroup $\left.\mathbb{Z}_{2}\left(\kappa_{1} \gamma^{2}\right)\right)$, as illustrated in Figure $5(\mathrm{~b})$.

\subsection{Bifurcation with nontrivial forced kernel symmetry}

We now discuss the (general) case in which $G$ acts nontrivially on the kernel $W_{2}$. The main difference with the case of trivial forced kernel symmetry is that $\tilde{f}_{1}(x)=0$ is a $G$-equivariant bifurcation 
problem with parameter symmetry, viewing - as before - $\tilde{f}_{1}$ as a family of endomorphisms of $W_{1}$ with parameters in $W_{2} \times \mathbb{R}^{\ell}$ :

$$
\tilde{f}_{1}\left(\rho_{1}(g) w_{1}, \rho_{2}(g) w_{2}, \lambda\right)=\rho_{1}(g) \tilde{f}_{1}\left(w_{1}, w_{2}, \lambda\right), \quad \forall g \in G .
$$

That is, in general, $\rho_{2}(g) \neq 1$.

Essentially, Lemma 3.8 is the main result showing the equivalence between the bifurcation problem $\tilde{f}_{1}=0$ on $K$ and a bifurcation problem with parameter symmetry: $\tilde{f}_{\text {red }}=0$. However, the computation of ker $d \pi_{1}(0,0)$ is not a straightforward exercise in the nontrivial forced kernel symmetry case and instead we consider the situation outlined below.

Consider the manifold $K$ at the organizing centre $(0,0)$ and we assume that the parameter space $\mathbb{R}^{\ell}$ satisfies $\ell \geq \operatorname{dim} W_{2}$. Let $k=\operatorname{dim} W_{2}$ and we write $\lambda=(\mu, \nu) \in \mathbb{R}^{k} \times \mathbb{R}^{\ell-k}$. In this case, $K$ is a $\operatorname{dim} V+(\ell-k)$ dimensional manifold. We make the following assumption:

(A1) There exists a parametrization of the manifold $K$ near $(0,0)$ of the form $\mu=\phi\left(w_{1}, w_{2}, \nu\right)$ where $\phi$ is a smooth $G$-invariant mapping.

Assumption (A1) is a special case of Proposition 6.4 which gives sufficient conditions for the existence of organizing centres and information about the structure of their parametrization. The examples found in this section are studied using the next result which is a rewriting of Lemma 3.8 in the above context.

Theorem 3.14 (Separable bifurcation with nontrivial forced kernel symmetry) Let $f$ : $V \times \mathbb{R}^{\ell} \rightarrow V$ be an $\ell$-parameter family of $G$-reversible equivariant vector fields with $\ell \geq \operatorname{dim} W_{2}$. Suppose that $f(0,0)=0,(0,0)$ has isotropy subgroup $G$ and is an organizing centre satisfying (A1). Suppose $f$ has a separable bifurcation at $(0,0)$. Let $B$ denote the set of equilibria of $f$ in a small neighbourhood of $(0,0)$.

Then, the following situation arises near an organizing centre: $B \subset V \times \mathbb{R}^{\ell}$ has a subset $B^{\prime}$ containing the origin with the following properties: $B^{\prime}$ is $G$-diffeomorphic to the bifurcation set of a smooth G-equivariant bifurcation problem with parameter symmetry

$$
h_{1}\left(\rho_{1}(g) w_{1}, \rho_{2}(g) w_{2}, \nu\right)=\rho_{1}(g) h_{1}\left(w_{1}, w_{2}, \nu\right), \quad \forall g \in G .
$$

on $W_{1}$, with parameters $\left(w_{2}, \nu\right) \in W_{2} \times \mathbb{R}^{\ell-k}$ with $k=\operatorname{dim} W_{2}$. In particular, all bifurcating branches tangent to $W_{1}$ at $(0,0)$ are contained in $B^{\prime}$.

Proof: For $(0,0)$ an organizing centre satisfying (A1), $K$ in $f_{2}^{-1}(0)$ is parametrized by $\mu=$ $\phi\left(w_{1}, w_{2}, \nu\right)$. Substituting into the $\tilde{f}_{1}$ equation we obtain

$$
h_{1}\left(w_{1}, w_{2}, \nu\right)=\tilde{f}_{1}\left(w_{1}, w_{2}, \phi\left(w_{1}, w_{2}, \nu\right), \nu\right)=0,
$$

with

$$
h_{1}\left(\rho_{1}(g) w_{1}, \rho_{2}(g) w_{2}, \nu\right)=\rho_{1}(g) g_{1}\left(w_{1}, w_{2}, \nu\right) .
$$

Since $\phi$ is smooth in $V$, then $h_{1}$ is also smooth and the zero set of $h_{1}$ is $G$-diffeomorphic to a subset $B^{\prime}$ of zeroes of $f$. 
Remark 3.15 In the nontrivial forced kernel symmetry case, our approach does not detect local solutions whose isotropy subgroup (of $G$ on $V$ ) is not an isotropy subgroup of $G$ on $W_{1}$. If such isotropy subgroups arise, an explicit computation of the nondegenerate local zero set is necessary.

We now illustrate these results with a few examples.

Example $3.16\left(\mathbb{Z}_{2}\right.$-reversible saddle-node in $\left.\mathbb{R}^{3} \times \mathbb{R}^{2}\right)$ Consider a $\mathbb{Z}_{2}$-reversible vector field in $\mathbb{R}^{3}$, where $\mathbb{Z}_{2}$ is generated by $R .(x, y, z)=(x,-y,-z)$. Consider a $\ell=2$ parameter family of $\mathbb{Z}_{2}$-reversible equivariant vector fields $f: W_{1} \oplus W_{2} \times \mathbb{R}^{2} \rightarrow \mathbb{R}^{3}$ with $f(0,0,0,0,0)=0$.

Each coordinate subspace is an irreducible representation of $G$ : the $y$-axis and $z$-axis are isomorphic irreducible representations, both $\sigma$-dual to the $x$-axis. Consequently, $W_{2}$ is a onedimensional subspace of $x=0$, and without loss of generality we choose $W_{2}=\{(x, y, z) \mid x=y=0\}$ and so $W_{1}=\{(x, y, z) \mid z=0\}$. Now, $s\left(\mathbb{Z}_{2}\right)=-1$ and $\mathbb{Z}_{2}$ acts as -1 (i.e. nontrivially) on $W_{2}$.

By definition of $W_{1}, f_{1}$ has a separable bifurcation at the origin if $\operatorname{ker}\left(d f_{1}\right)_{(0)}=\{(x, y) \mid y=0\}$. By Theorem 3.14, the set of equilibria $B^{\prime}$ is $G$-diffeomorphic to the zero set of a bifurcation problem with parameter symmetry $\tilde{h}_{1}(x, y, z, \mu)=0$ on $W_{1}$. We study a generic $\mathbb{Z}_{2}$-equivariant bifurcation problem with parameter symmetry $\tilde{h}_{1}: W_{1} \oplus W_{2} \times \mathbb{R}^{1} \rightarrow \mathbb{R}^{2}$ since $k=\operatorname{dim} W_{2}=1$.

Bifurcation problem with parameter symmetry: Using the Lyapunov-Schmidt reduction we solve $y$ in terms of $(x, z, \nu)$ to obtain the bifurcation equation in normal form $p(x, z, \nu)=$ $\nu+x^{2}+b z^{2}$ with $b= \pm 1$. As $\nu$ moves through 0 , there are two scenarios depending on the coefficient $b$ : an elliptic and a hyperbolic one. In the elliptic scenario $(b=+1)$, the family of zeroes with isotropy subgroup 1 near the bifurcation point forms an ellipse when $\nu<0$ disappearing when $\nu \geq 0$. In the hyperbolic scenario $(b=-1)$, the zero set with trivial isotropy subgroup forms a family of two hyperbola if $\nu \neq 0$ and a transversal intersection of two lines when $\nu=0$. This situation is illustrated in Figure 7 in a projection to a two-dimensional subspace.

Example $3.17\left(\mathbb{D}_{2}\right.$ symmetry in $\left.\mathbb{R}^{3} \times \mathbb{R}^{2}\right)$ Let $\mathbb{D}_{2}\left(R_{1}, R_{2}\right)$ act on $\mathbb{R}^{3}$ by

$$
R_{1} \cdot(x, y, z)=(x, y,-z) \quad \text { and } \quad R_{2} \cdot(x, y, z)=(-x,-y, z)
$$

and consider a two-parameter family of $\mathbb{D}_{2}$-reversible equivariant vector fields $f$ with a separable bifurcation at $(0,0,0,0,0)$.

The group $\mathbb{D}_{2}$ can arise as a reversing symmetry group in two ways:

$$
\text { (a) } \sigma\left(R_{2}\right)=\sigma\left(R_{1}\right)=-1 \quad \text { and } \quad(b) \quad \sigma\left(R_{2}\right)=-\sigma\left(R_{1}\right)=1 .
$$

The isotropy subgroup and index lattice for each type of reversibility is given in Figure 8. Case (b) naturally falls within the context of nonseparable bifurcations as any commuting linear map $L: \mathbb{R}^{3} \rightarrow \mathbb{R}^{3}$ is identically zero since there are no isomorphic representations in domain and range. Thus $W_{1}=\{0\}$ and case (b) cannot have separable bifurcations by definition.

We consider case (a) for which the kernel of a generic commuting linear map is one-dimensional. Each coordinate axis is an irreducible representation with the $x$ and $y$ axes isomorphic and nonisomorphic to the $z$-axis. We choose $W_{2}=\{(x, y, z) \mid y=z=0\}$. Therefore, $f$ has a separable bifurcation if $\operatorname{ker}(d f)_{(0,0,0,0,0)} \cap W_{1}=\{(y, z) \mid y=0\}$.

By Theorem 3.14, $B^{\prime}$ is $G$-diffeomorphic to the zero set of a $\mathbb{D}_{2}$-equivariant bifurcation problem with parameter symmetry $\tilde{h}_{1}(x, y, z, \nu)=0$ which we now study. 

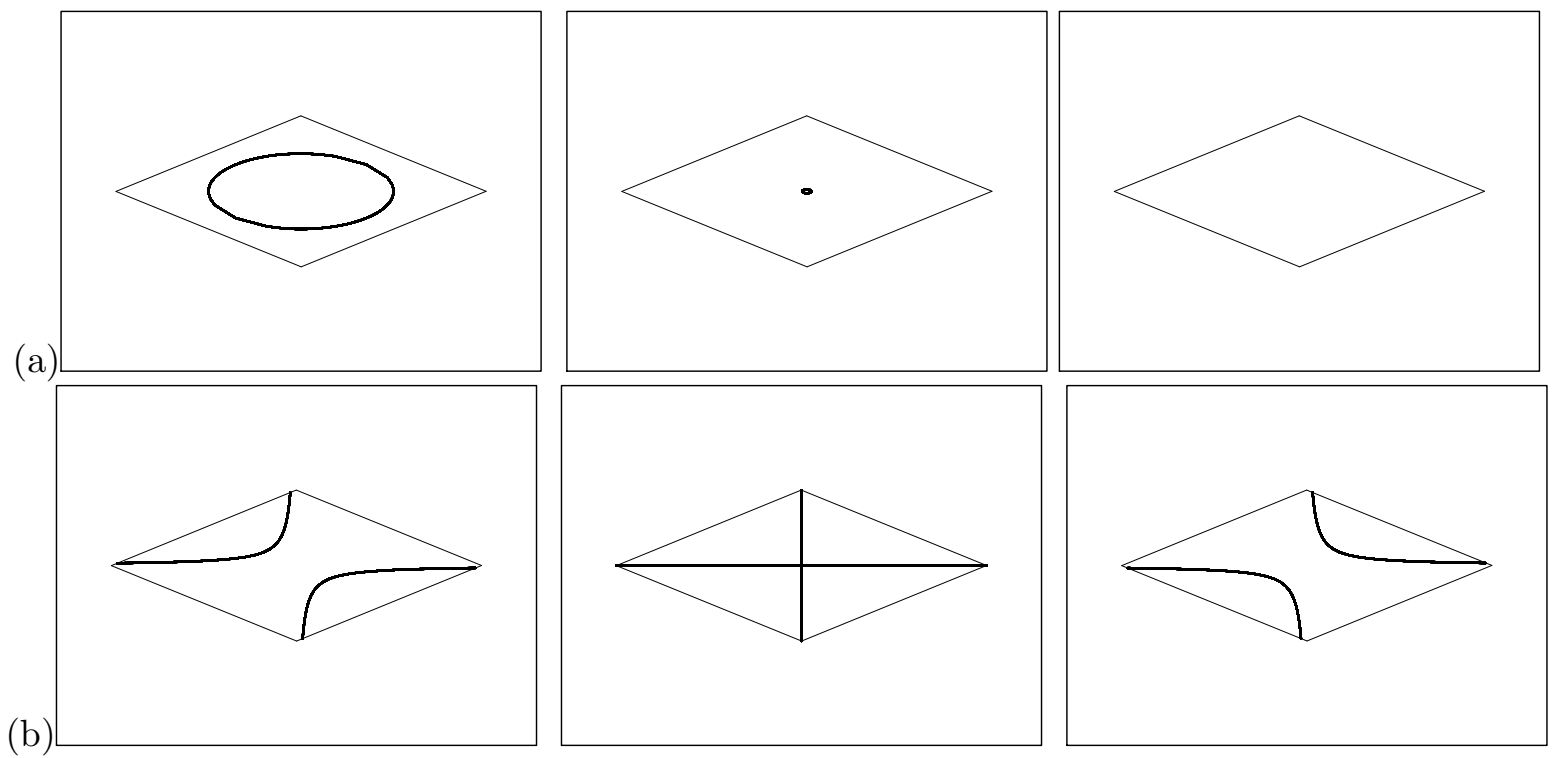

Figure 7: Generic codimension-one $\mathbb{Z}_{2}$ symmetry preserving turning point bifurcation in $W_{1} \oplus W_{2} \times$ $\mathbb{R}^{2}$ (see Example 3.16). Depicted are projections to a two-dimensional subspace. (a) elliptic case (b) hyperbolic case. Also bifurcation diagrams corresponding to Example 3.17 and Example 3.19.

Bifurcation problem with parameter symmetry: Since $W_{2}=\{(x, y, z) \mid y=z=0\}$ and the bifurcation direction is $z$, we use the Lyapunov-Schmidt reduction to solve $y$ as a function of $(x, z, \nu)$ and obtain a bifurcation equation $q\left(x^{2}, z^{2}, \nu\right) z=0$. For $z=0$ we obtain a two-dimensional manifold of solutions with isotropy subgroup $\mathbb{Z}_{2}\left(R_{1}\right)$. Since $q(0,0,0)=0$ at a separable bifurcation, and if $q_{\nu}(0,0,0) \neq 0$ is satisfied, then by the implicit function theorem we have a two-dimensional manifold of solutions which contains an open and dense set of solutions with isotropy subgroup 1, a one-dimensional submanifold of solutions with isotropy subgroup $\mathbb{Z}_{2}\left(R_{2}\right)$ and a one-dimensional submanifold of solutions with isotropy subgroup $\mathbb{Z}_{2}\left(R_{1}\right)$ (the intersection with the $z=0$ manifold).

Bifurcation problem with parameter symmetry: $\ell=1$ When one or more parameters are removed some branches of solution may disappear as this case shows. The bifurcation equation

(a)

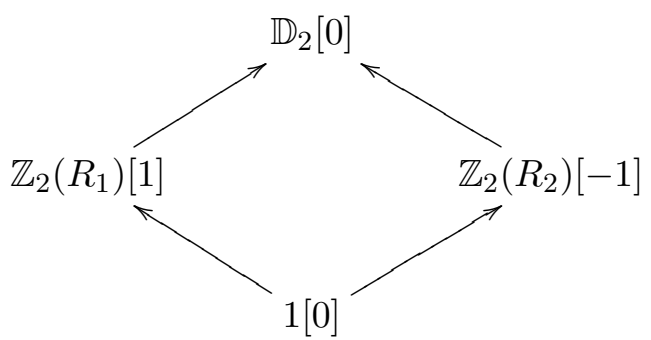

(b)

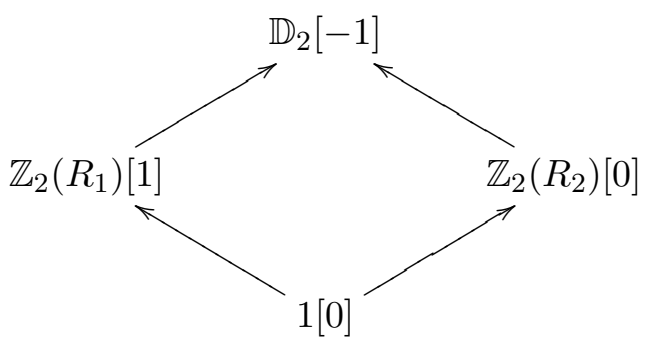

Figure 8: Isotropy subgroup and index lattice for the $\mathbb{D}_{2}$ action: (a) $\sigma\left(R_{2}\right)=\sigma\left(R_{1}\right)=-1$. (b) $\sigma\left(R_{2}\right)=-\sigma\left(R_{1}\right)=1$. 
(a)

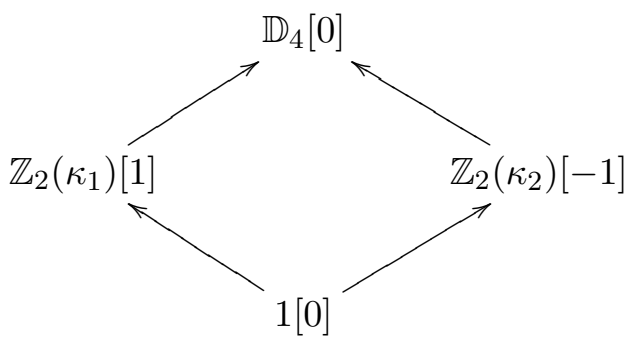

(b)

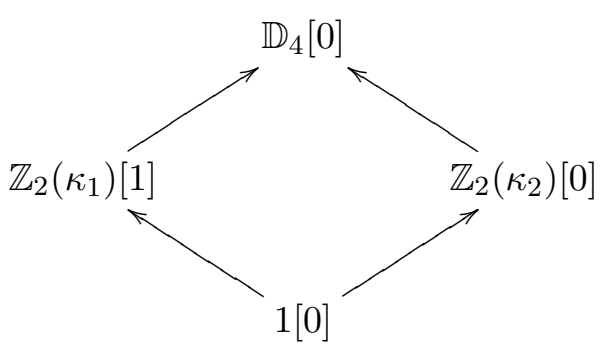

Figure 9: Isotropy subgroup and index lattice for (a) $\sigma\left(\kappa_{1}\right)=\sigma\left(\kappa_{2}\right)=-1$ (b) $\sigma\left(\kappa_{1}\right)=-\sigma\left(\kappa_{2}\right)=$ $-1$

$q=0$ has generic normal form $\epsilon\left(z^{2}+\delta x^{2}\right) z=0$ where $\epsilon= \pm 1$ and $\delta= \pm 1$, see Golubitsky and Schaeffer [18]. If $\delta=+1$, then no new solutions exist. If $\delta=-1$, we obtain a zero set of two lines, $z= \pm x$, crossing at the origin as seen in Figure 7 . The origin is the only equilibrium with isotropy subgroup $\mathbb{D}_{2}$ and there are 1-dimensional families of solutions with trivial isotropy. In particular, there are no solutions with isotropy subgroup $\mathbb{Z}_{2}\left(R_{2}\right)$.

Reversible-equivariant with $\ell=\mathbf{2}$. The bifurcating branch with isotropy subgroup $\mathbb{Z}_{2}\left(R_{1}\right)$ in fact is fattened by one dimension from two to three since $f_{2} \equiv 0$ on $\operatorname{Fix}\left(\mathbb{Z}_{2}\left(R_{1}\right)\right)$. The branches with isotropy subgroups $\mathbb{Z}_{2}\left(R_{2}\right)$ and 1 keep the same dimension in the full bifurcation problem. These dimensions correspond to $d_{\Sigma}$. A similar analysis applies for the $\ell=1$ case.

Example $3.18\left(\mathbb{D}_{4}\right.$ symmetry in $\left.\mathbb{R}^{3} \times \mathbb{R}^{2}\right)$ Consider the action of $\mathbb{D}_{4}$ on $\mathbb{R}^{3}$ generated by the reflections

$$
\kappa_{1} \cdot(z, x)=(\bar{z}, x) \quad \text { and } \quad \kappa_{2} \cdot(z, x)=(i \bar{z},-x) .
$$

where $z \in \mathbb{C}$ and $x \in \mathbb{R}$. We consider a two-parameter family of $\mathbb{D}_{4}$-reversible equivariant vector fields $f$. Here, $W_{1}=\mathbb{C}$ is a self-dual representation, $W_{2}=\mathbb{R}$.

We look at two cases of reversing symmetries:

$$
\sigma\left(\kappa_{1}\right)=\sigma\left(\kappa_{2}\right)=-1 \quad \text { and } \quad \sigma\left(\kappa_{1}\right)=-\sigma\left(\kappa_{2}\right)=-1 .
$$

The lattices of isotropy subgroups and indices are given in Figure 9.

The bifurcation equation splits into the system

$$
\begin{aligned}
& \tilde{f}_{1}(z, x, \lambda, \mu)=T f_{1}(z, x, \lambda, \mu)=0 \\
& f_{2}(z, x, \lambda, \mu)=0 .
\end{aligned}
$$

where $\tilde{f}_{1}: \mathbb{C} \times \mathbb{R} \times \mathbb{R}^{2} \rightarrow \mathbb{C}$ and $f_{2}: \mathbb{C} \times \mathbb{R} \times \mathbb{R}^{2} \rightarrow \mathbb{R}$.

By Theorem 3.14, there is a subset $B^{\prime}$ of $f^{-1}(0), G$-diffeomorphic to the zero set of a $\mathbb{D}_{4^{-}}$ equivariant steady-state bifurcation problem with parameter symmetry $h_{1}(z, x, \lambda)=0$. Furter et al. [15] study this bifurcation problem. In particular, we consider their solution to the generic case; see their Theorem 3.2.1 with $\epsilon_{5}=1$ and $m>1$ (the case $m<1$ is similar). For completeness, we describe this bifurcation diagram in details before returning to the original $\mathbb{D}_{4}$-reversible equivariant bifurcation problems. 

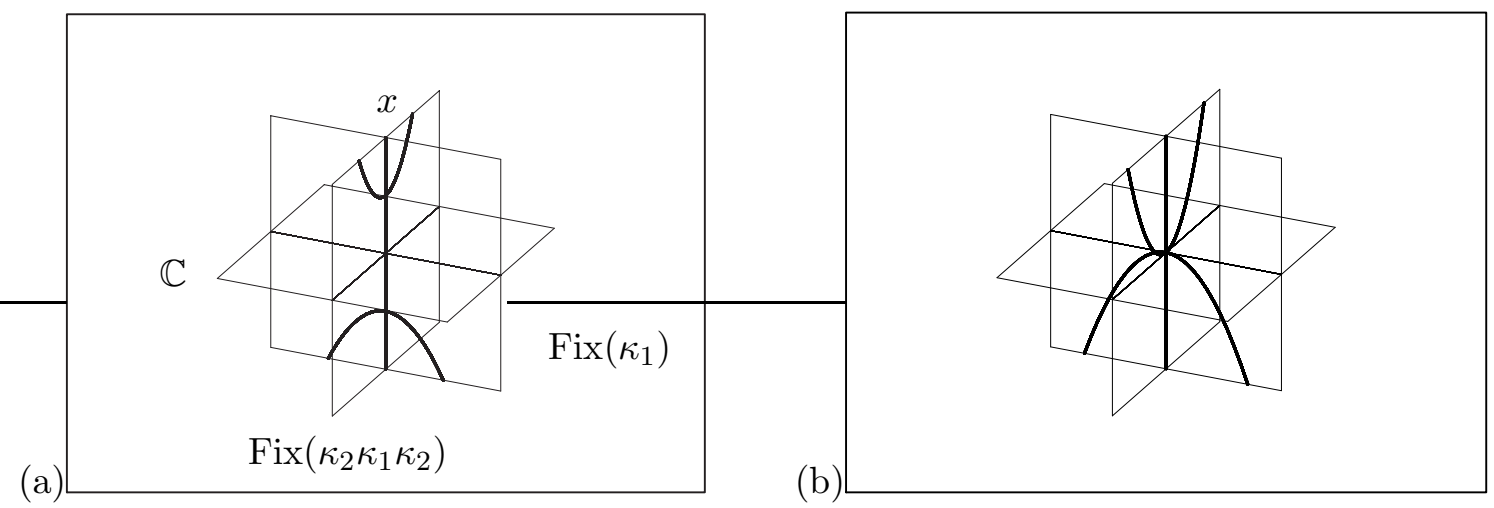

Figure 10: Bifurcation diagram of $\mathbb{Z}_{2}\left(\kappa_{1}\right)$ (and conjugate) symmetric solutions in a generic $\mathbb{D}_{4}$ bifurcation problem with parameter symmetry in $\mathbb{R}^{3}$ for (a) $\lambda<0$ and (b) $\lambda=0$.

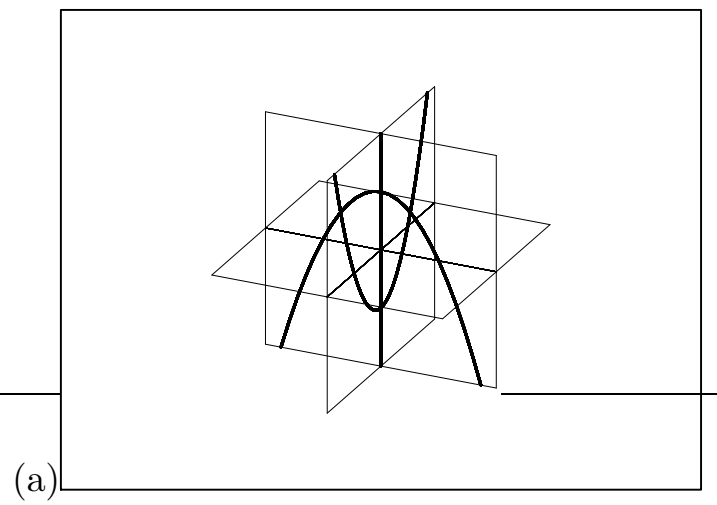

(b)

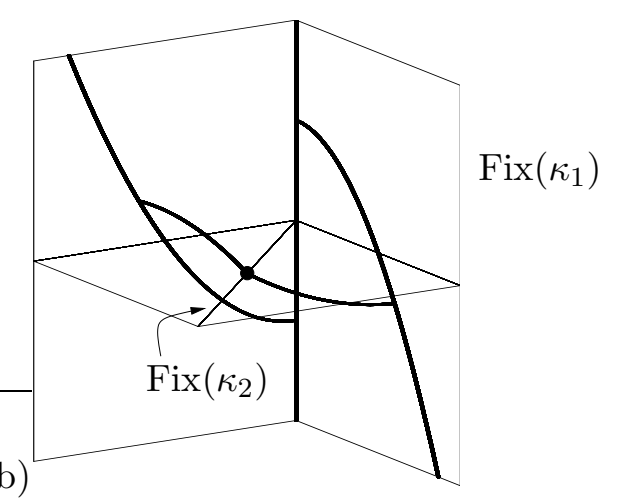

Figure 11: Bifurcating solutions for $\lambda>0$. (a) Solutions with isotropy subgroup $\mathbb{Z}_{2}\left(\kappa_{1}\right)$ (b) Detail of solutions with isotropy subgroup $\mathbb{Z}_{2}\left(\kappa_{2}\right)$ and 1 .

Bifurcation problem with parameter symmetry We consider the parameter $\lambda_{2}$ of Furter et al. [15] as a phase space variable since it corresponds to our variable $x$. We draw the bifurcation diagram of [15] with a three-dimensional phase space and one parameter instead of the twoparameter bifurcation problem with two-dimensional phase space of [15]. See Figure 10 and Figure 11. Solutions with isotropy subgroups $\mathbb{Z}_{2}\left(\kappa_{1}\right)$ are given in Figure 10 for $\lambda \leq 0$ and in Figure 11(a) for $\lambda>0$. For $\lambda \leq 0$ there are no solutions with isotropy subgroups $\mathbb{Z}_{2}\left(\kappa_{2}\right)$ and 1 . For $\lambda>0$, Figure 11(b) shows details of solutions with isotropy subgroups $\mathbb{Z}_{2}\left(\kappa_{1}\right), \mathbb{Z}_{2}\left(\kappa_{2}\right)$ and 1 in one quadrant of $\mathbb{R}^{3}$. The solutions in the other quadrants are obtained by applying the order four rotation symmetry $\kappa_{1} \kappa_{2}$. Thus in $\mathbb{R}^{3} \times \mathbb{R}$, solutions with isotropy subgroup $\mathbb{Z}_{2}\left(\kappa_{1}\right)$ form a twodimensional manifold, solutions with isotropy subgroup $\mathbb{Z}_{2}\left(\kappa_{2}\right)$ form a one-dimensional manifold and solutions with trivial isotropy subgroup form a two-dimensional manifold.

Reversible-equivariant case $\sigma\left(\kappa_{1}\right)=\sigma\left(\kappa_{2}\right)=-1$. The set $B^{\prime}$ contains the bifurcating branches for isotropy subgroups $\mathbb{Z}_{2}\left(\kappa_{1}\right), \mathbb{Z}_{2}\left(\kappa_{2}\right)$ and 1 described above. For isotropy subgroups $\mathbb{Z}_{2}\left(\kappa_{2}\right)$ and 1 , the dimension of the bifurcating branches is given by $d_{\Sigma}=s(\Sigma)+2$ in accordance with Proposition 2.3 , but not for isotropy subgroup $\mathbb{Z}_{2}\left(\kappa_{1}\right)$. Now, note that $f_{2} \equiv 0$ on 
$\operatorname{Fix}_{V}\left(\mathbb{Z}_{2}\left(\kappa_{1}\right)\right)$, therefore, the dimension of the bifurcating branch with isotropy subgroup $\mathbb{Z}_{2}\left(\kappa_{1}\right)$ is 3 which corresponds to $d_{\Sigma}=s(\Sigma)+2$. Thus, $B^{\prime}$ contains all bifurcating branches given, up to $G$-diffeomorphism, by the bifurcation diagram of the $\mathbb{D}_{4}$-equivariant steady-state bifurcation problem with parameter symmetry studied in [15].

Reversible-equivariant case $\sigma\left(\kappa_{1}\right)=-\sigma\left(\kappa_{2}\right)=-1$. Again, $B^{\prime}$ contains the bifurcating branches found above in the bifurcation problem with parameter symmetry. However, the dimension of the branches may differ as we explain now. Note that $f_{2} \equiv 0$ on $\operatorname{Fix}\left(\mathbb{Z}_{2}\left(\kappa_{i}\right)\right)$ for $i=1,2$ since $\sigma\left(\kappa_{1}\right)$ and $\sigma\left(\kappa_{2}\right)$ act on $W_{2, \sigma}=\mathbb{R}$ by -1 . Hence, the bifurcating branches of $h_{1}=0$ for isotropy subgroups $\mathbb{Z}_{2}\left(\kappa_{1}\right)$ and $\mathbb{Z}_{2}\left(\kappa_{2}\right)$ are also branches for the full bifurcation problem with an additionnal dimension for each since the second parameter $\mu$ extends the zero sets trivially. The branch with trivial isotropy subgroup remains unchanged as a two-dimensional branch and the dimensions of the three branches are given by $d_{\Sigma}=s(\Sigma)+2$.

Alternate case: $\sigma\left(\kappa_{1}\right)=-\sigma\left(\kappa_{2}\right)=-1$ Suppose that $f$ only depends on one parameter instead of two. Using Theorem 3.14 we would only find the branch with isotropy subgroup $\mathbb{Z}_{2}\left(\kappa_{1}\right)$. An organizing centre is obtained as $x=\phi(z, \lambda)$ where $\phi$ is a smooth map. Substituting we obtain the equation $\tilde{f}_{1}(z, \phi(z, \lambda), \lambda)=0$ which is a one parameter $\mathbb{D}_{4}$-equivariant bifurcation problem (with no parameter symmetry). Typically, we expect one dimensional branches of equilibria with isotropy subgroup $\mathbb{Z}_{2}\left(\kappa_{1}\right)$ and $\mathbb{Z}_{2}\left(\kappa_{2}\right)$. Note that the branch with isotropy subgroup $\mathbb{Z}_{2}\left(\kappa_{1}\right)$ has an extra dimension since $s\left(\mathbb{Z}_{2}\left(\kappa_{1}\right)\right)=1$.

The alternate case of the last example shows that the minimal number of parameters to obtain all the bifurcating branches may be lower than the number necessary for our approach based on organizing centres and Theorem 3.14 to work. Moreover, the equivariant bifurcation problem may not even involve parameter symmetry. However, obtaining a sharp bound on the number of parameters and determining if there is parameter symmetry or not might depend on performing explicit calculations on the $f_{2}=0$ equation. Rather than aiming to be efficient with parameters and bifurcation equations, in this paper we have given preference to the presentation of a method that is generally applicable and relies only on calculations of $G$-equivariant bifurcation problems with parameter symmetry.

\subsection{Nonseparable bifurcation}

Nonseparability of $f$ at a branching point is induced by the fact that ker $d_{V} f$ contains more copies of an irreducible representation of $G$ than present in the forced kernel. Therefore, the bifurcation can lead to a topological change in the nondegenerate local zero set. It is not our aim to discuss these bifurcations in detail here. We provide just one example as an illustration.

Example 3.19 (nonseparable bifurcation for $\mathbb{Z}_{2}$ in $\mathbb{R}^{3}$ ) Let $G=\mathbb{Z}_{2}(R)$ and $V=\mathbb{R}^{3}$ be as in Example 2.1. Suppose that $f_{\lambda}(x, y, z)$ is a one-parameter family of $\mathbb{Z}_{2}(R)$-reversible equivariant vector fields with $f_{0}(0,0,0)=0$, so that $\operatorname{ker}\left(d f_{0}\right)_{(0,0,0)}=\{(x, y, z) \mid z=0\}$ and $\operatorname{ker}\left(d f_{\lambda}\right)_{(x, y, z)}$ has minimal dimension for $\lambda$ close to 0 and $(x, y, z)$ in a small neighbourhood of $(0,0,0)$.

By Lyapunov-Schmidt reduction to $\operatorname{ker}\left(d f_{0}\right)_{(0,0,0)}=\operatorname{Fix}_{V}\left(\mathbb{Z}_{2}(R)\right)$, we are led to look for equilibria with isotropy subgroup $\mathbb{Z}_{2}(R)$ that are zeroes of

$$
f_{\mathbb{Z}_{2}}: \operatorname{Fix}_{V}\left(\mathbb{Z}_{2}(R)\right) \times \mathbb{R} \rightarrow \operatorname{Fix}_{V_{\sigma}}\left(\mathbb{Z}_{2}(R)\right)
$$


where $\operatorname{Fix}_{V_{\sigma}}\left(\mathbb{Z}_{2}(R)\right)=\{(x, y, z) \mid x=y=0\}$ is one-dimensional. From the notation in Example 2.1 we have

$$
f_{\mathbb{Z}_{2}}(x, y, \lambda)=r_{\lambda}(x, y, 0)
$$

where $r_{0}(0,0,0)=0$ and $d r(0,0,0)=0$ from the kernel condition. Generically, zeroes of this function are determined by the zeroes of the normal form $a x^{2}+b y^{2}+c \lambda$ with $a, b \neq 0$.

At $\lambda=0$, the zero set of $f_{\mathbb{Z}_{2}}$ is locally a point if $a, b$ have the same sign (elliptic case) and the intersection of two one-dimensional curves if $a, b$ have different signs (hyperbolic case). The unfoldings of these singularities at $\lambda=0$ are illustrated by the sketches of the equilibria in $\mathbb{R}^{3}$ of Figure 7 where the plane corresponds to $\operatorname{Fix}_{V}\left(\mathbb{Z}_{2}(R)\right)$.

\section{Branching and bifurcation of relative equilibria and (relative) periodic solutions}

In the earlier discussion, we have focussed on symmetric equilibria that are fixed by $G$. In particular, the main hypothesis that we have relied on is that the reference equilibrium does not lie on a continuous group orbit of equilibria. In that case the relevant group action in the neighbourhood of the equilibrium is the action of the isotropy subgroup of the equilibrium.

An equilibrium that lies on a continuous group orbit of equilibria is an example of a relative equilibrium. In the Section 4.1 we discuss how branching and bifurcation of such equilibria and more generally relative equilibria can be understood from our results on bifurcation and branching of equilibria.

Our results furthermore apply (via reduction) to a range of other local bifurcation problems, including nonHopf bifurcation and branching of periodic and relative periodic solutions. We briefly discuss this in Section 4.2.

\subsection{Relative equilibria}

We consider a smooth $G$-reversible equivariant vector field $f$ on a manifold $\mathcal{M}$, with $G$ acting properly. A solution $x(t) \in \mathcal{M}$, with $x(0)=x$, is called a relative equilibrium if for all $t, x(t) \in G x$, where the group orbit $G x$ is defined as

$$
G x:=\{y \in \mathcal{M} \mid y=g . x, \forall g \in G\} .
$$

Hence, such a solution is an equilibrium for the induced vector field on the quotient space $\mathcal{M} / G$

Relative equilibria, and their bifurcations, have received much attention in the literature. Of particular relevance to the discussion here is Lamb and Wulff's treatment of relative equilibria in reversible equivariant systems [27]. We recall some of their results.

Let $x \in \mathcal{M}$ be a point on a relative equilibrium with isotropy subgroup $\Sigma \leq G$, ie $\Sigma x=x$, implying that for $s \in \Sigma, s . x(t)=x(\sigma(s) t)$ for all $t$. Then we may consider a $\Sigma$-invariant slice $V$ through $x$, that is transversal to $G x$. $V$ locally has the structure of a linear vector space, and evidently $\operatorname{dim} G-\operatorname{dim} \Sigma+\operatorname{dim} V=\operatorname{dim} \mathcal{M}$. 
The slice may be used to define a tubular neighbourhood $W$ of $G x$, by taking the direct product of $V$ and $G$, and quotienting out the $\Sigma$-action on $V$ :

$$
W \simeq \frac{V \times G}{\Sigma}
$$

One may lift $W, \Sigma$-equivariantly, to $V \times G$, so that the vector field in $W$ can now be described in $V \times G$ coordinates. Now, let $(v, g) \in V \times G$, then one obtains the following form for the differential equation on $V \times G$ :

$$
\left\{\begin{array}{l}
\frac{d v}{d t}=f_{V}(v), \\
\frac{d g}{d t}=g f_{G}(v) .
\end{array}\right.
$$

Here, $f_{V}$ is a $\Sigma$-reversible equivariant vector field on $V$ : for all $s \in \Sigma$

$$
f_{V}(\rho(s) v)=\rho_{\sigma}(s) f_{V}(v), \forall v \in V
$$

where $\rho$ is the representation of $\Sigma$ on $V$, and $\rho_{\sigma}$ its dual. $f_{G}$ is also $\Sigma$-reversible equivariant: for all $s \in \Sigma$

$$
f_{G}(\rho(s) v)=\sigma(s) \operatorname{Ad}_{\sigma} f_{G}(v), \forall(v, g) \in V \times G,
$$

where $\operatorname{Ad}_{\sigma}$ is the adjoint action of $\Sigma$ on $L G$, the Lie algebra of $G$.

It is important to note that the equations (13) form a skew product: the vector field $f_{V}$ on the slice $V$ does not depend on the group-coordinate $g \in G$, but meanwhile drives the motion on the group orbit, with $f_{G}$ depending on $v \in V$.

A group orbit of equilibria corresponds to a relative equilibrium $G(v, 0)$ with $f_{V}(v)=f_{G}(v)=$ 0 . In this case, one also says that the relative equilibrium has no drift. A general relative equilibrium $G(v, 0)$ satisfies $f_{V}(v)=0$. If $f_{G}(v) \neq 0$ one says that the relative equilibrium drifts along the $G$-group orbit.

From the skew-product equations, it is evident that our results for equilibria in $\Sigma$-reversible equivariant vector fields on $V$, also apply to relative equilibria with isotropy subgroup $\Sigma$ and slice $V$. In particular, results about the nondegenerate local zero set of Section 2 and bifurcation theorems such as Theorem 3.9 and Theorem 3.14 apply also to relative equilibria with isotropy subgroup $\Sigma$ and slice $V$, reading "relative equilibria" wherever statements concern "equilibria".

The main thrust of this paper has been to describe the set of equilibria in parameter families of reversible equivariant vector fields. This leads us to digress somewhat on the drift of relative equilibria. In order to know whether a relative equilibrium consists of group orbits of equilibria we need to determine whether there is a flow (drift) along group orbits, or not.

We now recall a result on drift of reversible equivariant relative equilibria by Lamb and Wulff [27]. But first, we introduce some notation. Let $L G_{\sigma}$ denote the Lie algebra $L G$ of $G$ with an action of $\Sigma$, defined by

$$
\gamma \cdot \xi=\operatorname{Ad}_{\gamma}^{\sigma}(\xi)=\sigma(\gamma) \operatorname{Ad}_{\gamma}(\xi), \quad \xi \in L G_{\sigma}, \gamma \in \Sigma .
$$

This action is called the $\sigma$-adjoint action of $\Sigma$. Correspondingly,

$$
\operatorname{Fix}_{L G_{\sigma}} \Sigma:=\left\{\xi \in L G_{\sigma} \mid \gamma \cdot \xi=\xi, \forall \gamma \in \Sigma\right\}
$$


Theorem 4.1 (Drift of reversible equivariant relative equilibria [27]) Let Gx be a relative equilibrium for a $G$-reversible equivariant vector field in $\mathcal{M}$, and let $\Sigma$ denote the isotropy subgroup of $x$. Then, generically, the relative equilibrium drifts along a maximal reversible torus of dimension

$$
n_{\Sigma}^{\sigma}=\operatorname{dim} \operatorname{Fix}_{L G_{\sigma}} \Sigma-\operatorname{dim} \operatorname{Fix}_{L \Sigma_{\sigma}} \Sigma .
$$

We now obtain an alternative description of the drift dimension above in terms of a $\sigma$-index. For this, we define a $\sigma$-index of $\Sigma$ on the orbit $G x$ as follows. Let

$$
\operatorname{Fix}_{G}(\Sigma):=\left\{g \in G \mid s g s^{-1}=g, \forall s \in \Sigma\right\}
$$

then

$$
s_{G / \Sigma}(\Sigma):=\left[\operatorname{dim} \operatorname{Fix}_{G}(\Sigma)-\operatorname{dim}_{F_{i x}}(\Sigma)\right]-\left[\operatorname{dim}_{F_{i x}}(\Sigma)-\operatorname{dim} \operatorname{Fix}_{L \Sigma_{\Sigma}}(\Sigma)\right] .
$$

Now, recall that

$$
n_{\Sigma}=\operatorname{dim}_{\operatorname{Fix}_{G}}(\Sigma)-\operatorname{dim} \operatorname{Fix}_{\Sigma}(\Sigma)
$$

and the following result follows directly.

Theorem 4.2 (Generic drift dimension) Let $n_{\Sigma}^{\sigma}$ be as defined in Theorem 4.1 then

$$
n_{\Sigma}^{\sigma}=n_{\Sigma}-s_{G / \Sigma}(\Sigma)
$$

The new formula for $n_{\Sigma}^{\sigma}$ shows that $s_{G / \Sigma}(\Sigma)$ provides a correction to the generic drift in the non-reversible case.

We do not aim to provide general results on bifurcation from group orbits of equilibria (and relative equilibria in general), but rather illustrate the use of the bundle equations.

Example $4.3\left(\mathbb{Z}_{2}\right.$-reversible pitchfork bifurcation of relative equilibria) Consider now a $\mathbf{S O}(2) \times \mathbb{Z}_{2}$-reversible equivariant vector field in $\mathbb{R}^{3} \times \mathbb{R}$ as discussed in Example 2.5. Suppose we have $\left(x_{0}, 0,0\right) \in \mathbb{R}^{3}$, with $x_{0} \neq 0$, lying on a one-dimensional $\mathbf{S O}(2)$-group orbit of equilibria. Then $V$ is a two-dimensional slice transversal to the $\mathbf{S O}(2)$-group orbit, which may be chosen as a subset of the plane $x=x_{0}$ and $R$ acts on $V$ as $R .(y, z)=(y,-z) . d f_{V}$ has no forced kernel and by Theorem 3.2 the local "slice"-steady-state bifurcation problem of the relative equilibrium in $V$ reduces to that of a steady-state bifurcation of a $\mathbb{Z}_{2}$-equivariant vector field. In a one-parameter family, on $V$ we may hence observe a generic $\mathbb{Z}_{2}$-symmetry breaking pitchfork bifurcation of equilibria.

For the full flow this implies that there is a $\mathbb{Z}_{2}$-symmetry breaking pitchfork bifurcation of relative equilibria. If we consider the flow on the group orbit, the $\mathbb{Z}_{2}$-invariant relative equilibrium is a one-dimensional family of equilibria $\left(d_{\mathbb{Z}_{2}}=n_{\mathbb{Z}_{2}}=1\right)$, but the bifurcating relative equilibria without symmetry generically do not consist of equilibria but are flow-invariant $\mathbf{S O}(2)$-group orbits with a nonzero drift $\left(d_{1}-n_{1}=0-1=-1<0\right)$. 


\subsection{Bifurcation and branching of (relative) periodic solutions}

We can use the results of this paper to understand so-called nonHopf branching and bifurcation from periodic solutions in reversible equivariant systems. NonHopf bifurcations are bifurcations arising when Floquet multipliers go through finite (resonant) roots of unity [23, 24].

The main observation [24] is that the bifurcation and branching equations of nonHopf type always formally reduce to reversible equivariant steady-state bifurcation equations. The fact that one may conclude the true existence of solutions from the formal (normal form) analysis is guaranteed by a general result on finite determinacy for such bifurcation problems by Field [10]. Here we merely discuss an elementary application and we do not digress on the reduction, but for more details see [24].

Example 4.4 (Subharmonic branching of periodic solutions) Consider a vector field $f$ in $W=\mathbb{R}^{4}$ that is $\mathbb{Z}_{2}$-reversible, where the time-reversal symmetry acts as $R .(x, y, u, v)=(x, y,-u,-v)$. As $s\left(\mathbb{Z}_{2}\right)=0$, generically equilibria with isotropy subgroup $\mathbb{Z}_{2}$ are isolated.

Now, suppose we have a $\mathbb{Z}_{2}$-symmetric periodic solution, ie a solution $x(t)=x(t+T)$ for all $t(T>0)$, satisfying for all $t$

$$
x(t)=R \cdot x(-t) .
$$

We can construct a Poincare section $V$ for this periodic solution, so that the dynamics near the periodic solution may be represented by a Poincaré map $F: V \rightarrow V$. We study the map using a formal normal form, which is associated to a $\mathbb{Z}_{2}$-reversible vector field. We denote this vector field $f_{V}$. Now, $d f_{V}$ has a one-dimensional forced kernel, as $s_{V}\left(\mathbb{Z}_{2}\right)=1$, even though $s_{W}\left(\mathbb{Z}_{2}\right)=0$.

Hence, away from a branching point, we observe a one-dimensional family of $\mathbb{Z}_{2}$-symmetric equilibria in the normal form vector field. In turn, this branch of equilibria corresponds to a onedimensional family of fixed points for the Poincaré return map $F$. In $V$, this family corresponds to a one-dimensional family of periodic solutions with slowly varying period.

Somewhere on the one-dimensional branch of periodic solutions we may encounter a periodic solution with Floquet multipliers $\{\exp (2 \pi i p / q), \exp (-2 \pi i p / q), 1\}$. These Floquet multipliers lead to a $\mathbb{D}_{q}=\mathbb{Z}_{q} \rtimes \mathbb{Z}_{2}$-reversible equivariant normal from vector field, the equivariance originating as a formal symmetry induced by the linear part $d F$ of the Poincaré return map $F$. The $\mathbb{D}_{q^{-}}$ reversible equivariant normal form vector field displays steady-state branching as discussed in Example 3.17 for the case $q=2$, and in fact for general $q$ as the bifurcation set of a codimension one steady-state $\mathbb{D}_{q}$-equivariant bifuration problem (as discussed for instance in [19]), with $2 q$ $\mathbb{Z}_{2}$-invariant branches of equilibria bifurcating from the main $\mathbb{D}_{q}$-invariant branch. For the return map, the resulting branches correspond to families of symmetric period $q$ orbits coming from the one-dimensional family of fixed points with $\mathbb{Z}_{2}$-symmetry. In $V$ one observes branches of $q$-tupled symmetric periodic solutions from a one-dimensional family of symmetric periodic solutions.

The above approach provides a proof of the generic occurrence of reversible subharmonic branching, alternative to those of Vanderbauwhede [37, 39] and Ciocci [4]. Using the same approach one can also study subharmonic branching in reversible equivariant vector fields.

The pattern of subharmonic branching along a one-parameter family of periodic solutions and subsequent subharmonic branching from the branches yields an intricate structure, that is sometimes referred to as the subharmonic branching tree [39, 33]. Roberts and Lamb [33] studied 
the period-doubling part of such a branching tree numerically in various examples of reversible maps in $\mathbb{R}^{3}$, and observed that such a period-doubling branching tree has self-similar properties, with scaling factors similar to those observed in period-doubling cascades of one-parameter families of area-preserving maps in $\mathbb{R}^{2}$.

Our results can also be applied to describe local nonHopf bifurcations of relative periodic solutions as these also essentially reduce to reversible equivariant steady-state bifurcation problems $[41,24]$.

\section{$5 \quad$ Linear reversible equivariant systems}

In this section we discuss some results on linear reversible equivariant maps, based on the treatment in [26]. Recall the definitions of the representation $\rho$ and its $\sigma$-dual $\rho_{\sigma}$ of $G$, from (2). We say that $\rho$ and $\rho_{\sigma}$ are $(\sigma-) d u a l$ representations. It is easy to verify that if $\rho$ is irreducible, then so is $\rho_{\sigma}$. A representation of $G$ on $V$ has an isotypic decomposition

$$
V=V_{1} \oplus \cdots \oplus V_{m}
$$

where $V_{j}$ is a direct sum of $G$-isomorphic irreducible representations. It is well known (by Schur's lemma) that $G$-equivariant maps respect this isotypic decomposition. However $G$-reversible equivariant maps map an isotypic component $V_{j}$ to the isotypic component of its $\sigma$-dual, which we denote by $V_{j, \sigma}$. From the isotypic components $V_{j}$ we therefore construct the $\sigma$-isotypic components $\widehat{V}_{j}=V_{j}+V_{j, \sigma}$ which are left invariant by $G$-reversible equivariant maps. Note that $\widehat{V}_{j}=V_{j}$ if $V_{j}$ is a direct sum of self-dual irreducible representations and $\widehat{V}_{j}=V_{j} \oplus V_{j, \sigma}$ otherwise. Accordingly, we obtain a $\sigma$-isotypic decomposition

$$
V=\widehat{V_{1}} \oplus \cdots \oplus \widehat{V_{\hat{m}}}
$$

which is respected by $G$-reversible equivariant maps.

Lamb and Roberts [26] classify the different types of reversible equivariant linear maps on $\sigma$ isotypic components. In particular they show that there are three types of non-self-dual irreducible representations and seven types of self-dual irreducible representations. They also show that an irreducible representation $V$ is self-dual if and only if there exists a linear $G$-reversible equivariant map $T: V \rightarrow V$ satisfying $T^{2}= \pm 1$.

The question is whether the existence of non-self-dual irreducible representations prevents the existence of an invertible reversible equivariant map. It turns out that the answer depends on whether the dimensions of the isotypic components $V_{j}$ and $V_{j, \sigma}$ inside the $\sigma$-isotypic component $\widehat{V}_{j}$ are the same or not.

Lemma 5.1 Let $\widehat{V}_{j}=V_{j} \oplus V_{j, \sigma}$ be a $\sigma$-isotypic component corresponding to non-self-dual irreducible representation of $G$. Let $T: \widehat{V}_{j} \rightarrow \widehat{V}_{j}$ be a linear $G$-reversible equivariant map. Then, $\operatorname{dim} \operatorname{ker} T \geq\left|\operatorname{dim} V_{j}-\operatorname{dim} V_{j, \sigma}\right|$. Moreover, there exists a $T$ attaining the lower bound for the kernel. 
Proof: Without loss of generality suppose $\operatorname{dim} V_{j} \geq \operatorname{dim} V_{j, \sigma}$. Recall that $T: V_{j} \rightarrow V_{j, \sigma}$ and $T: V_{j, \sigma} \rightarrow V_{j}$. Hence, the restriction $\left.T\right|_{V_{j}}$ has a kernel of dimension greater or equal than $\operatorname{dim} V_{j}-\operatorname{dim} V_{j, \sigma}$. The restriction $\left.T\right|_{V_{j, \sigma}}$ does not give rise to a forced kernel. To construct a $T$ with minimal kernel, decompose $V_{j}=U_{j} \oplus K_{j}$, where $U_{j}, K_{j}$ are $G$-invariant and $\operatorname{dim} U_{j}=\operatorname{dim} V_{j, \sigma}$. Take coordinates $(u, k, v) \in U_{j} \oplus K_{j} \oplus V_{j, \sigma}$. Then $T(u, k, v)=(v, k, u)$ is invertible, $G$-reversible equivariant and has $\operatorname{dim} \operatorname{ker} T=\operatorname{dim} V_{j}-\operatorname{dim} V_{j, \sigma}$.

Corollary 5.2 Let $\widehat{V}_{j}=V_{j} \oplus V_{j, \sigma}$ be a $\sigma$-isotypic component corresponding to non-self-dual irreducible representation of $G$. Then there exists an invertible $G$-reversible equivariant map $T: \widehat{V}_{j} \rightarrow \widehat{V}_{j}$ if and only if $\operatorname{dim} V_{j}=\operatorname{dim} V_{j, \sigma}$.

Using the results above we can now formulate a precise definition of the forced kernels introduced in Section 3.

Definition 5.3 Let $\widehat{V}_{j}=V_{j} \oplus V_{j, \sigma}$ be a $\sigma$-isotypic component for a nonself-dual representation of $G$ and let $T_{j}: \widehat{V}_{j} \rightarrow \widehat{V}_{j}$ be a linear $G$-reversible equivariant map attaining the lower bound $\operatorname{dim} \operatorname{ker} T_{j}=\left|\operatorname{dim} V_{j}-\operatorname{dim} V_{j, \sigma}\right|>0$. We call the isomorphism class of the representation of $G$ on $\operatorname{ker} T_{j}$ the forced kernel for $\widehat{V}_{j}$. The forced kernel for $V$ is the direct sum of the forced kernels for its $\sigma$-isotypic components $\widehat{V}_{j}$.

The following proposition shows that near a separable bifurcation point in a family of reversible equivariant maps the forced kernel can always be taken to be independent of the parameter values.

Proposition 5.4 Suppose that $L(\lambda)$ is a parametrized family of linear reversible equivariant maps. Suppose that ker $L(0) \supseteq W_{2}$ where $W_{2}$ is in the isomorphism class of the forced kernel, and that the decompositions of $W_{2}$ and its complement in $\operatorname{ker} L(0)$ into irreducible representations have no isomorphic components. Then there exists a linear change of coordinates, depending smoothly on $\lambda$ such that $W_{2} \subseteq \operatorname{ker} L(\lambda)$ for all sufficiently small values of $\lambda$.

Proof: Recall that the irreducible representations that enter the forced kernel are determined up to isomorphism by the representation of the group. Since ker $L(0) \backslash W_{2}$ has no irreducible representations isomorphic to irreducible representations in $W_{2}$, then for sufficiently small $\lambda$, ker $L(\lambda)$ contains a forced kernel $W_{2}(\lambda), G$-isomorphic to $W_{2}$, so that ker $L(\lambda) \backslash W_{2}(\lambda)$ contains no irreducible representations of $G$ that are isomorphic to irreducible representations in $W_{2}(\lambda)$.

We follow in detail the argument in case $W_{2}$ contains copies of only one irreducible representation. Let $\hat{V}_{j}=V_{j} \oplus V_{j, \sigma}$ be the corresponding $\sigma$-isotypic component of $V$. On $\hat{V}, L(\lambda)$ has the form $[26]$

$$
L(\lambda)=\left[\begin{array}{cc}
0 & A_{n \times m}(\lambda) \\
B_{m \times n}(\lambda) & 0
\end{array}\right]
$$

where $A_{n \times m}(\lambda)$ and $B_{m \times n}(\lambda)$ are $n \times m$ and $m \times n$ matrices whose entries depend smoothly on $\lambda$. Without loss of generality, we may assume that $n>m$ and $\operatorname{so} \operatorname{dim} W_{2}=\left|\operatorname{dim} V_{j}-\operatorname{dim} V_{j, \sigma}\right|=$ $n-m$. Therefore, $W_{2}(\lambda)=\operatorname{ker} B_{m \times n}(\lambda)$. Hence, $B_{m \times n}(0)$ is surjective, and by the implicit function theorem $W_{2}(\lambda)$ depends smoothly on $\lambda$, as the basis vectors of $W_{2}(\lambda)$ do. Consequently, there exists a $G$-equivariant linear map $P(\lambda)$ depending smoothly on $\lambda$ mapping $W_{2, \lambda}$ to $W_{2}$. 
If $W_{2}$ contains irreducible representations of more than one $\sigma$-isotypic component of $V$, the same argument holds for each $\sigma$-isotypic component, and can be applied sequentially, as $L(\lambda)$ respects the $\sigma$-isotypic decomposition of $V$.

\section{$6 \quad$ Existence of Organizing Centres}

In this section we state and prove two results on the existence of organizing centres, Theorem 6.4 and Theorem 6.10. These organizing centre are persistent to small perturbations in $f_{2}$. These results are found in the later subsections and require some notation and results from $G$-transversality theory. In the first subsection, we introduce the notation and concepts needed for the remainder.

\subsection{G-transversality}

Let $V$ and $W$ be $G$-representations and $f: V \rightarrow W$ a smooth $G$-equivariant map. If $G$ acts trivially on $V$ and $W$ the problem of finding generic solutions to an equation $f=0$ can be expressed in terms of transversality conditions. Generically smooth maps are transverse to 0, which means that at a point $x$ with $f(x)=0$ the derivative $d f(x)$ must be surjective. If $\operatorname{dim} V<\operatorname{dim} W$ then derivatives can not be surjective and so zero-sets are generically empty. If $\operatorname{dim} V \geq \operatorname{dim} W$ then the implicit function theorem implies that, near most points, zero sets are smooth manifolds [20].

Suppose now that $G$ acts nontrivially on $V$ and $W$. Then there may not be any surjective $G$-equivariant linear maps from $V$ to $W$ even if $\operatorname{dim} V \geq \operatorname{dim} W$. However if, for example, $\operatorname{Fix}_{W}(G)=\{0\}$ then the zero set is forced to be non-empty. It follows that transversality to $\{0\}$ in $W$ is no longer a generic property. To overcome problems of this nature, Bierstone [1] and Field [6] each developed equivariant transversality theories. Field later proved that these theories are equivalent [7]. We use the notation and approach of [1].

Denote by $C_{G}^{\infty}(V, W)$ the space of $G$-equivariant $C^{\infty}$ maps from $V$ to $W$. By work of Schwarz and Poénaru [34, 31, 19] on smooth invariant theory there exists a minimal set of homogeneous polynomial generators $\left\{F_{1}, \ldots, F_{k}\right\}$ for $C_{G}^{\infty}(V, W)$. That is, any $f \in C_{G}^{\infty}(V, W)$ can be written as

$$
f(x)=\sum_{i=1}^{k} h_{i}(x) F_{i}(x)
$$

where $h_{i}: V \rightarrow \mathbb{R}$ are $G$-invariant smooth functions.

Consider the map $F: V \times \mathbb{R}^{k} \rightarrow W$ defined by $F(x, t)=\sum_{i=1}^{k} t_{i} F_{i}(x)$ and the set $\mathcal{E}=F^{-1}(0)$. Without loss of generality, we can assume that $\operatorname{Fix}_{V}(G)=\{0\}$ and $\operatorname{Fix}_{W}(G)=\{0\}$ since the trivial representations of $G$ in $V$ can be considered as parameters and the trivial representations of $G$ in $W$ give rise to constant homogeneous generators which can be factored from the problem of finding $\mathcal{E}$, see Field [11].

Define $\operatorname{graph}_{f}: V \rightarrow V \times \mathbb{R}^{k}$ by $\operatorname{graph}_{f}(x)=\left(x, h_{1}(x), \ldots, h_{k}(x)\right)$. The following result is straightforward.

Lemma 6.1 Let $f \in C_{G}^{\infty}(V, W)$. Then, 
(1) $f=F \circ g_{r a p h}$

(2) $f^{-1}(0)=\operatorname{graph}_{f}^{-1}(\mathcal{E})$.

Thus the zero set of any function $f$ is the intersection of graph ${ }_{f}$ with the algebraic variety $\mathcal{E}$. The set $\mathcal{E}$ is called the universal zero set and $F$ is the universal map.

Let $\Sigma$ be an isotropy subgroup. Recall that the conjugacy class of $\Sigma$ is called the isotropy type and is denoted by $(\Sigma)$.

Let $V_{(\Sigma)}=\left\{x \in V \mid G_{x} \in(\Sigma)\right\}$. Consider the sets

$$
\mathcal{E}_{(\Sigma)}=\left\{(x, t) \in \mathcal{E} \mid x \in V_{(\Sigma)}\right\}
$$

over all isotropy types $(\Sigma)$. The set $\mathcal{E}$ is the disjoint union of the sets $\mathcal{E}_{(\Sigma)}$. In particular, from the fact that $\operatorname{Fix}_{V}(G)=\{0\}$, we have $\mathcal{E}_{G}=\mathbb{R}^{k}$.

Before we define $G$-transversality we need to review some basic facts about stratifications of sets. See Field $[12,9,11]$ for clear and concise introductions to stratifications and Gibson et al [16] for a more detailed and general description.

Stratifications A stratification $S$ of a subset $X$ of $\mathbb{R}^{n}$ is a locally finite partition of $X$ into smooth, connected submanifolds of $\mathbb{R}^{n}$ called strata. In particular, if $X$ is semialgebraic (ie defined by polynomial equations and inequalities), then $\mathcal{S}$ is a semialgebraic stratification if each stratum is semialgebraic. A stratification is said to be Whitney regular if it satisfies the Whitney regularity conditions (see for example [9] or [16] for full descriptions). Of particular interest to us is the frontier condition of Whitney regular stratifications: if $A, B$ are strata satisfying $\bar{A} \cap B \neq \emptyset$ then $B \subset \bar{A}$. An important consequence of Whitney regularity in our context is the following. If $\bar{A} \cap B \neq \emptyset$, then any map transverse to $B$ is also transverse to $A$ near $B$.

Let $X_{i}$ be the union of all strata of dimension less or equal than $i$. A Whitney regular stratification $S$ of a set $X$ is said to be canonical if for each $i, S_{i}$ is the largest smooth submanifold of $X_{i}$ for which $S_{j}$ is Whitney regular over $S_{i}$, for all $j>i$.

Every semialgebraic set admits a canonical Whitney regular stratification [16]. The following result relates the canonical Whitney regular stratification of $\mathcal{E}$ to the isotropy type submanifolds $\mathcal{E}_{(\Sigma)}$

Theorem 6.2 (Field $[\mathbf{1 2}, \mathbf{1 1}])$ The canonical semialgebraic Whitney regular stratification $\mathcal{S}$ of $\mathcal{E}$ induces a semialgebraic Whitney stratification of each $\mathcal{E}_{(\Sigma)}$.

That is, $\mathcal{E}_{(\Sigma)} \cap \mathcal{S}$ determines uniquely a stratification $\mathcal{S}_{(\Sigma)}$ of $\mathcal{E}_{(\Sigma)}$ into $G$-invariant strata, see Field [11] for details. For strata of $\mathcal{S}_{(\Sigma)}$ we use the notation $S_{(\Sigma)}^{i}$ to denote a strata of dimension $i$. To simplify the notation in what follows we use $(G)=G$ and $(\Sigma):=e$ if $\Sigma$ is the identity subgroup of $G$.

We now return to the definition of the equivariant transversality condition we require for equivariant maps $f: V \rightarrow W$.

Definition 6.3 ( $G$-transversality) The $G$-equivariant map $f$ is $G$-transversal to $0 \in W$ at $0 \in V$ if graph $_{f}: V \rightarrow V \times \mathbb{R}^{k}$ is transverse to the canonical semialgebraic Whitney regular stratification of $\mathcal{E}$. 
The characteristic transversality, openness, density and isotopy theorems continue to hold for $G$-transversality though the transversality isotopy theorem only holds up to homeomorphism. We refer to [1] and [6] for details and precise statements of results.

\subsection{Persistent Manifolds at Organizing centres}

In this section, we present a sufficient condition for the existence of organizing centres valid for all compact group actions. For the study of separable bifurcations we assume that $(0,0) \in V \times \mathbb{R}^{\ell}$ is an equilibrium with isotropy subgroup $G$. We use the splitting $V=W_{1} \oplus W_{2}$ where, invoking Proposition 5.4, $W_{2}$ is the forced kernel for all values of the parameter.

Let $\left\{F_{1}, \ldots, F_{q}\right\}$ be a minimal set of homogeneous generators for $C_{G}^{\infty}\left(V, W_{2, \sigma}\right)$. Then

$$
f_{2}\left(w_{1}, w_{2}, \lambda\right)=\sum_{i=1}^{q} p_{i}\left(w_{1}, w_{2}, \lambda\right) F_{i}\left(w_{1}, w_{2}\right)
$$

where $p_{i}: V \times \mathbb{R}^{\ell} \rightarrow \mathbb{R}$ are $G$-invariant functions. The next proposition gives sufficient condition for the existence of organizing centres.

Theorem 6.4 Let $\left\{F_{1}\left(w_{1}, w_{2}\right), \ldots, F_{q}\left(w_{1}, w_{2}\right)\right\}$ be a minimal set of homogeneous polynomial generators for $C_{G}^{\infty}\left(V, W_{2, \sigma}\right), f_{2}\left(w_{1}, w_{2}, \lambda\right)=\sum_{i=1}^{q} p_{i}\left(w_{1}, w_{2}, \lambda\right) F_{i}\left(w_{1}, w_{2}\right)$ and let $y=\left(w_{2}, \lambda\right) \in$ $W_{2} \times \mathbb{R}^{\ell}$. If $p_{i}(0,0,0)=0$ for all $i=1, \ldots, q$ and the mapping $P: V \times \mathbb{R}^{\ell} \rightarrow \mathbb{R}^{q}$ defined by

$$
P\left(w_{1}, y\right)=\left(p_{1}\left(w_{1}, y\right), \ldots, p_{q}\left(w_{1}, y\right)\right)^{t}
$$

is such that $\left(d_{y} P\right)_{(0,0)}$ is onto, then there exists a smooth $G$-invariant manifold $K \subset f_{2}^{-1}(0)$ of dimension $\operatorname{dim} V+(\ell-q)$ containing $\left(w_{1}, y\right)=(0,0)$. The restriction of $\pi_{1}$ maps $K$ submersively to $W_{1}$ at $(0,0)$. Furthermore, $K$ is stable to small perturbations of $f_{2}$.

Proof: The Implicit Function Theorem guarantees that $K=P^{-1}(0)$ is a smooth $\operatorname{dim} V+(\ell-q)$ dimensional manifold obtained by solving $q$ of the variables in $W_{2} \times \mathbb{R}^{\ell}$ as a function of $w_{1}$ and the remaining $\operatorname{dim} W_{2}+(\ell-q)$ variables. By definition of $K$, the projection $\pi_{1}$ restricted to $K$ is a submersion at $(0,0)$. The stability of $\mathrm{K}$ follows from the genericity of the transversality condition implied by the requirement that $\left(d_{y} P\right)_{(0,0)}$ is surjective.

Remark 6.5 The assumption (A1) in Section 3.4 follows from the conclusions of Theorem 6.4 when the $q$ coordinates in $W_{2} \times \mathbb{R}^{\ell}$ are parameters.

The next example shows that the sufficient condition for the existence of a smooth manifold $K$ found in Theorem 6.4 is sometimes also necessary.

Example 6.6 Let $V=\mathbb{R}^{4} \times \mathbb{R}^{2}$ with coordinates $(x, y)$ and $G=\mathbb{Z}_{2}(R)$ where $R$. $(x, y)=(x,-y)$. We split $V=W_{1} \oplus W_{2}$ where $\operatorname{dim} W_{1}=4, \operatorname{dim} W_{2}=2$ and $\left.R\right|_{W_{2}}=+I$. Then, $f_{2}: V \rightarrow W_{2, \sigma}$ is

$$
f_{2}(x, y)=\left[p_{i j}(x, y, \lambda)\right] y
$$


where $\left[p_{i j}(x, y, \lambda)\right]$ is a $2 \times 2$ matrix of $\mathbb{Z}_{2}$-invariant functions, $y=\left(y_{1}, y_{2}\right)^{t}$ and $\lambda \in \mathbb{R}^{\ell}$. The universal map is given by

$$
\hat{f}_{2}(x, y, t):=\left[t_{i j}\right] y
$$

where $\left[t_{i j}\right]$ is a $2 \times 2$ real matrix. Let $\mathcal{E}$ be the universal zero set of $\hat{f}_{2}$, then $\mathcal{E}=\mathcal{E}_{G} \cup \mathcal{E}_{e}$ where

$$
\mathcal{E}_{G}=\{(x, y, t) \mid y=0\}, \quad \text { and } \quad \mathcal{E}_{e}=\left\{(x, y, t) \mid\left[t_{i j}\right] y=0, y \neq 0\right\} .
$$

The semialgebraic sets $\mathcal{E}_{G}$ and $\mathcal{E}_{e}$ are stratified by rank of the matrix $\left[t_{i j}\right]$ (recall that the superscript denotes the dimension of the stratum):

$$
\begin{aligned}
& S_{G}^{4}=\left\{(x, y, t) \mid \operatorname{rank}\left[t_{i j}\right]=0, y=0\right\}, \quad S_{G}^{7}=\left\{(x, y, t) \mid \operatorname{rank}\left[t_{i j}\right]=1, y=0\right\} \\
& S_{G}^{8}=\left\{(x, y, t) \mid \operatorname{rank}\left[t_{i j}\right]=2, y=0\right\}
\end{aligned}
$$

and

$$
S_{e}^{6}=\left\{(x, y, t) \mid \operatorname{rank}\left[t_{i j}\right]=0, y \neq 0\right\}, \quad S_{e}^{9}=\left\{(x, y, t) \mid\left[t_{i j}\right] y=0, \operatorname{rank}\left[t_{i j}\right]=1, y \neq 0\right\} .
$$

The relationship between the strata are shown in Figure 6.6 where the direction of the arrow shows inclusion in the closure of the strata.

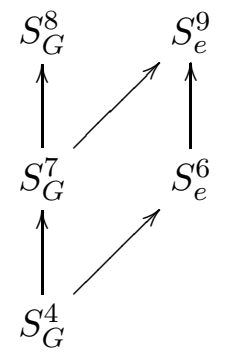

Figure 12: Inclusion diagram for strata of $\mathcal{E}$.

For $(x, y, \lambda)=(0,0,0)$ to be an organizing centre, the closure of the subset of $f_{2}^{-1}(0)$ with isotropy subgroup 1 must project to a full neighborhood of the origin in $W_{1}$. Now, the rank $\left[t_{i j}\right]=1$ condition in $S_{e}^{9}$ forces a relationship of the form $y_{1}=m y_{2}$. Thus, if graph $f_{2}(0,0,0)$ intersects $S_{e}^{9}$ transversally, the closure of $\operatorname{graph}_{f_{2}}^{-1}\left(S_{e}^{9}\right)$ does not project to a full neighborhood of the origin in $W_{1}$ and this is true no matter how many parameters we add to the problem.

We see that $S_{e}^{6}$ is the only stratum of $\mathcal{E}_{e}$ such that the closure of $S_{e}^{6}$ projects to a full neighborhood of $W_{1}$. So, in order for $(x, y)=(0,0)$ to be an organizing centre, with $K$ given by the inverse image of a stratum, then graph $f_{2}(0,0)$ has to intersect the stratum $S_{G}^{4}$ since it is the only one which lies in the boundary of the stratum $S_{e}^{6}$. This means that we must have $p_{i j}(0,0,0)=0$ for $i, j=1,2$ and with the transversality condition we are in the context of Theorem 6.4.

In the following example a weaker condition than the one given in Proposition 6.4 guarantees the existence of a smooth manifold $K$ at an organizing centre. 
Example 6.7 (Example 3.18) The group $G=\mathbb{D}_{4}$ acts on $\mathbb{C} \times \mathbb{R}$ as $\kappa_{1} \cdot(z, x)=(\bar{z}, x)$ and $\kappa_{2} \cdot(z, x)=(i \bar{z},-x)$. Recall that $W_{1}=\mathbb{C}$. In the case, $\sigma\left(\kappa_{1}\right)=-\sigma\left(\kappa_{2}\right)=-1$, we have $f_{2}(z, x, \lambda)=\left(p\left(x^{2},|z|^{2}, z^{4}+\bar{z}^{4}, x\left(z^{2}+\bar{z}^{2}\right), \lambda\right) x+q\left(x^{2},|z|^{2}, z^{4}+\bar{z}^{4}, x\left(z^{2}+\bar{z}^{2}\right), \lambda\right)\left(z^{2}+\bar{z}^{2}\right)\right)\left(z^{2}-\bar{z}^{2}\right)$. If $p(0,0,0,0) \neq 0$ (which is generically satisfied), one can solve the equation

$$
p\left(x^{2},|z|^{2}, z^{4}+\bar{z}^{4}, x\left(z^{2}+\bar{z}^{2}\right), \lambda\right) x+q\left(x^{2},|z|^{2}, z^{4}+\bar{z}^{4}, x\left(z^{2}+\bar{z}^{2}\right), \lambda\right)\left(z^{2}+\bar{z}^{2}\right)=0
$$

near $(x, z, \lambda)=(0,0,0)$ using the implicit function theorem and the smooth manifold $K$ is given by

$$
x=\phi\left(|z|^{2}, z^{4}+\bar{z}^{4}, \lambda\right)\left(z^{2}+\bar{z}^{2}\right) .
$$

It is clear that $\pi_{1}(K)=W_{1}$ and so $(x, z, \lambda)=(0,0,0)$ is an organizing centre. Note that the parameter $\lambda$ does not play any role in finding $K$.

Note that the manifold $K$ in this case is obtained directly from the implicit function theorem applied to $f_{2}$. One can possibly write conditions on the representation of $G$ on $V$ which would guarantee the existence of an organizing centre by a direct application of the implicit function theorem on $f_{2}$. We do not pursue this question further in this work.

We now present another construction of organizing centre where the manifold $K$ is structurally stable. We still assume that $V$ and $W$ have no trivial representations. Consider the projection $\pi_{1}: W_{1} \times W_{2} \times \mathbb{R}^{q} \rightarrow W_{1}$.

Definition 6.8 We say that a submanifold $S \subset \mathcal{E}_{e}$ covers $W_{1}$ if $\pi_{1}(\bar{S})$ contains an open neighborhood of the origin in $W_{1}$.

Consider now $S_{G}^{\prime}:=\mathcal{E}_{G} \cap S^{\prime}$ for some stratum $S^{\prime}$ of the canonical stratification of $\mathcal{E}$ and let $S_{G}$ be a stratum of $S_{G}^{\prime}$.

Definition 6.9 The stratum $S_{G}$ is central if there exists a submanifold $S \subset \mathcal{E}_{e}$ covering $W_{1}$ and $S_{G} \subset \bar{S} \cap \mathbb{R}^{q}$.

We now construct a particular type of central strata. To do this, we first suppose that the principal orbit type of $W_{1}$ is the trivial subgroup and we let $\hat{f}_{2}$ be the universal map for $C_{G}^{\infty}\left(V, W_{2, \sigma}\right)$. Consider

$$
\hat{f}_{2}\left(w_{1}, 0, t\right)=\sum_{i=1}^{q} t_{i} F_{i}\left(w_{1}, 0\right) .
$$

We define a submanifold $S \subset \mathcal{E}_{e}$ as follows

$$
S:=\left\{\left(w_{1}, 0, t\right) \in \mathcal{E}_{e} \mid t_{i}=0 \quad \text { if } \quad F_{i}\left(w_{1}, 0\right) \not \equiv 0\right\} .
$$

Therefore, $S$ covers $W_{1}$ and let $S_{G}$ be the largest stratum of $\mathcal{E}_{G}$ contained in $\bar{S} \cap \mathbb{R}^{q}$. Since $S_{G}$ is a semialgebraic subset of $\mathbb{R}^{q}$, we can write it as

$$
S_{G}=\left\{t \in \mathbb{R}^{q} \mid \eta_{i}(t)=0, \zeta_{j}(t)>0 \quad i=1, \ldots, r \quad \text { and } \quad j=1, \ldots, s\right\}
$$


where $\eta_{i}$ and $\zeta_{j}$ are polynomials. In particular, some of the equalities in the definition of $S_{G}$ include the defining conditions of for $S$; that is, $t_{i}=0$ for all $i$ for which $F_{i}\left(w_{1}, 0\right) \not \equiv 0$.

Then codim $S_{G}=r$ and we define the following mapping

$$
\mathcal{P}\left(w_{1}, \lambda\right)=\left(\eta_{1}\left(P\left(w_{1}, 0, \lambda\right)\right), \ldots, \eta_{r}\left(P\left(w_{1}, 0, \lambda\right)\right)\right)^{t}
$$

where $P\left(w_{1}, w_{2}, \lambda\right)$ is defined in Theorem 6.4.

Theorem 6.10 Suppose that the principal isotropy type of $W_{1}$ is the identity subgroup and graph $_{f_{2}}(0,0)$ intersects a central stratum $S_{G}$ defined as above. If $\left(d_{\lambda} \mathcal{P}\right)_{(0,0)}$ is onto, then there exists a smooth $G$-invariant manifold $K \subset f_{2}^{-1}(0)$ of dimension $\operatorname{dim} W_{1}+\ell-r$ containing the origin in $V \times \mathbb{R}^{\ell}$. Furthermore, the restriction of $\pi_{1}$ maps $K$ submersively to $W_{1}$ at $(0,0) \in V \times \mathbb{R}^{\ell}$ and $K$ is stable to small perturbations of $f_{2}$.

Proof: $\quad$ Since $\operatorname{graph}_{f_{2}}(0,0)$ intersects $S_{G}$, then $\mathcal{P}(0,0)=0 \in \mathbb{R}^{r}$ and $\zeta_{j}(P(0,0))>0$ for all $j=1, \ldots, s$. Because $\left(d_{\lambda} \mathcal{P}\right)_{(0,0)}$ is onto, the implicit function theorem guarantees the existence of a smooth mapping $\phi$ where $r$ parameters in $\lambda$ are written as a function of $w_{1}$ and remaining parameters in $\lambda$. The mapping $\phi$ defines a smooth submanifold $K$ of dimension $\operatorname{dim} W_{1}+\ell-r$ containing the origin in $V \times \mathbb{R}^{\ell}$ such that $\mathcal{P}(K) \equiv 0$ and which also satisfies $\zeta_{j}(P(K))>0$ for $j=1, \ldots, s$. By definition of $S_{G}, K \subset f_{2}^{-1}(0)$ and $G$-invariance of $K$ comes from the $G$-invariance of $\mathcal{P}$. By construction of $K, \pi_{1}$ restricted to $K$ is a submersion at $(0,0)$.

Remark 6.11 The nondegeneracy condition on $\left(d_{\lambda} \mathcal{P}\right)_{(0,0)}$ implies automatically that $f_{2}$ is $G$ transverse to $0 \in W_{2, \sigma}$ at $(0,0) \in V \times \mathbb{R}^{\ell}$. This can be seen easily by noticing that $\operatorname{rank}\left(d_{\lambda} \mathcal{P}\right)_{(0,0)}=$ $r$ implies that the column vectors of $\left(d_{\lambda} P\right)_{(0,0)}$ must span a subspace of dimension $r$ complementary to $T_{\operatorname{graph}_{f_{2}}(0,0)} S_{G}$ and thus guaranteeing $G$-transversality.

Remark 6.12 Suppose that a $G$-reversible-equivariant bifurcation problem $f(x, \lambda)=0$ has an organizing centre at $\left(x_{0}, \lambda_{0}\right)$ such that the manifold $K$ has the structure given by Theorem 6.10. Because $w_{2}=0$, the reduced bifurcation problem $\tilde{f}_{\text {red }}: W_{1} \times \mathbb{R}^{\ell-r} \rightarrow W_{1}$ is a $G$-equivariant bifurcation problem (with no parameter symmetry)

We now conclude by finding all the possible ways of obtaining an organizing centre in Example 3.18 and in particular we show organizing centres as given by Theorem 6.10 for the two cases of reversing symmetries. We begin by revisiting Example 6.7 and look at the structure of the strata in $\mathcal{E}$. Recall that $W_{1}=\mathbb{C}$ in these examples.

Example 6.13 The group $G=\mathbb{D}_{4}$ acts on $\mathbb{C} \times \mathbb{R}$ as $\kappa_{1} \cdot(z, x)=(\bar{z}, x)$ and $\kappa_{2} \cdot(z, x)=(i \bar{z},-x)$. In the case, $\sigma\left(\kappa_{1}\right)=-\sigma\left(\kappa_{2}\right)=-1$, we have

$$
f_{2}(z, x, \lambda)=\left(p(x, z, \lambda) x+q(x, z, \lambda)\left(z^{2}+\bar{z}^{2}\right)\right)\left(z^{2}-\bar{z}^{2}\right)
$$

where $p$ and $q$ are $\mathbb{D}_{4}$-invariant functions of $x, z$ and $\lambda$. So

$$
\hat{f}_{2}\left(z, x, t_{1}, t_{2}\right)=t_{1} x\left(z^{2}-\bar{z}^{2}\right)+t_{2}\left(z^{2}+\bar{z}^{2}\right)\left(z^{2}-\bar{z}^{2}\right) .
$$


The zero set $\mathcal{E}$ is decomposed as follows in terms of representatives of isotropy types $(\Sigma)$ :

$$
\begin{aligned}
\mathcal{E}_{\mathbb{D}_{4}} & =\left\{\left(z, x, t_{1}, t_{2}\right) \mid z=x=0\right\}, \\
\mathcal{E}_{\left(\mathbb{D}_{2}\right)} & =\left\{\left(z, x, t_{1}, t_{2}\right) \mid z=0, x \neq 0\right\} \\
\mathcal{E}_{\left(\mathbb{Z}_{2}\left(\kappa_{1}\right)\right)}=\left\{\left(z, x, t_{1}, t_{2}\right) \mid z= \pm \bar{z}, z \neq 0\right\}, & \mathcal{E}_{\left(\mathbb{Z}_{2}\left(\kappa_{2}\right)\right)}=\left\{\left(z, x, t_{1}, t_{2}\right) \mid z= \pm i \bar{z}, z \neq 0, x=0\right\}, \\
\mathcal{E}_{e} & =\left\{\left(z, x, t_{1}, t_{2}\right) \mid t_{1} x+t_{2}\left(z^{2}+\bar{z}^{2}\right)=0, z \neq 0, z \neq \pm \bar{z}, \text { and } z \neq \pm i \bar{z} \text { or } x \neq 0\right\} .
\end{aligned}
$$

The manifold $\mathcal{E}_{e}$ has four strata

$$
\begin{aligned}
& S_{e}^{3}=\left\{\left(z, x, t_{1}, t_{2}\right) \in \mathcal{E}_{e} \mid t_{1}=t_{2}=0\right\}, \quad S_{e}^{\prime 3}=\left\{\left(z, x, t_{1}, t_{2}\right) \in \mathcal{E}_{e} \mid t_{2}=0, t_{1} \neq 0, x=0\right\} \\
& S_{e}^{\prime \prime 3}=\left\{\left(z, x, t_{1}, t_{2}\right) \in \mathcal{E}_{e} \mid t_{1}=0, t_{2} \neq 0, z= \pm i \bar{z}, z \neq 0, x \neq 0\right\} \\
& S_{e}^{5}=\left\{\left(z, x, t_{1}, t_{2}\right) \in \mathcal{E}_{e} \mid t_{1} \neq 0, t_{2} \neq 0, x=\frac{-t_{2}\left(z^{2}+\bar{z}^{2}\right)}{t_{1}}\right\} .
\end{aligned}
$$

The strata $S_{e}^{3}, S_{e}^{\prime 3}$ and $S_{e}^{5}$ cover $W_{1}$ while $S_{e}^{\prime \prime 3}$ does not because of the condition $z= \pm i \bar{z}$. Note that the stratum $S_{e}^{\prime 3}$ defines a manifold as in (14), but not the other strata. The stratification of $\mathcal{E}_{G}$ is given by

$$
\begin{aligned}
& S_{G}^{0}=\overline{S_{e}^{3}} \cap \mathbb{R}^{2}=\left\{\left(0,0, t_{1}, t_{2}\right) \mid t_{1}=t_{2}=0\right\}, \quad S_{G}^{1}=\overline{S_{e}^{\prime \prime 3}} \cap \mathbb{R}^{2}=\left\{\left(0,0, t_{1}, t_{2}\right) \mid t_{1}=0, t_{2} \neq 0\right\} \\
& S_{G}^{\prime 1}=\overline{S_{e}^{\prime 3}} \cap \mathbb{R}^{2}=\left\{\left(0,0, t_{1}, t_{2}\right) \mid t_{2}=0, t_{1} \neq 0\right\}, \quad S_{G}^{2}=\overline{S_{e}^{5}} \cap \mathbb{R}^{2}=\left\{\left(0,0, t_{1}, t_{2}\right) \mid t_{1} \neq 0, t_{2} \neq 0\right\}
\end{aligned}
$$

where $S_{G}^{0}, S_{G}^{\prime 1}$ and $S_{G}^{2}$ are all central strata while $S_{G}^{1}$ is not.

We can obtain the manifold $K$ at an organizing centre in three ways depending on which central strata graph $f_{2}(0,0)$ intersects in $\mathcal{E}_{\mathbb{D}_{4}}: S_{G}^{0}, S_{G}^{\prime 1}$ or $S_{G}^{2}$. The case where graph $f_{2}(0,0)$ intersects $S_{G}^{2}$ is treated in Example 6.7. If graph $f_{2}(0,0)$ intersects $S_{G}^{0}$, this corresponds to the situation described in Theorem 6.4 and the mapping defining $K$ is

$$
P(z, x, \lambda)=(p(x, z, \lambda), q(x, z, \lambda)) .
$$

In order to satisfy the transversality condition of Theorem 6.4 , we suppose that $\lambda \in \mathbb{R}^{2}$ and we must have that $\left(d_{\lambda} P\right)_{(0,0,0)}$ is nonsingular.

Finally, if $\operatorname{graph}_{f_{2}}(0,0)$ intersects $S_{G}^{\prime}$, this corresponds to the context of Theorem 6.10 and the mapping defining $K$ is

$$
\mathcal{P}(z, \lambda)=q(0, z, \lambda) .
$$

The transversality condition in Theorem 6.10 is

$$
\left(d_{\lambda} \mathcal{P}\right)_{(0,0)}=\frac{\partial q}{\partial \lambda}(0,0,0) \neq 0
$$

and from the implicit function theorem, there exists a smooth $G$-invariant mapping $\phi: \mathbb{C} \rightarrow \mathbb{R}$ with $\phi(0)=0$ such that $\mathcal{P}(z, \phi(z)) \equiv 0$. Thus, $K$ is a two-dimensional manifold defined near $(z, x, \lambda)=(0,0,0)$ by $\{(z, x, \lambda) \in V \times \mathbb{R} \mid x=0, \lambda=\phi(z)\}$. 
We now look at the second case of reversing symmetry in Example 3.18. The following case is similar to the previous example, but it also shows that a manifold which covers $W_{1}$ does not necessary lead to a manifold $K$ at an organizing centre.

Example 6.14 (Example 3.18) Consider again $\mathbb{D}_{4}$ acting on $\mathbb{C} \times \mathbb{R}$ as $\kappa_{1} \cdot(z, x)=(\bar{z}, x)$ and $\kappa_{2} \cdot(z, x)=(i \bar{z},-x)$, but we now consider the case $\sigma\left(\kappa_{1}\right)=\sigma\left(\kappa_{2}\right)=-1$. We have

$$
f_{2}(z, x, \lambda)=\left(p(x, z, \lambda)+q(x, z, \lambda)\left(z^{2}+\bar{z}^{2}\right) x\right)\left(z^{2}-\bar{z}^{2}\right)
$$

where again $p$ and $q$ are $\mathbb{D}_{4}$-invariant functions of $x, z$ and $\lambda$. So, $\hat{f}_{2}\left(z, x, t_{1}, t_{2}\right)=\left(t_{1}+t_{2} x\left(z^{2}+\right.\right.$ $\left.\left.\bar{z}^{2}\right)\right)\left(z^{2}-\bar{z}^{2}\right)$ and $\mathcal{E}$ decomposes in terms of isotropy types:

$$
\begin{aligned}
\mathcal{E}_{\mathbb{D}_{4}} & =\left\{\left(z, x, t_{1}, t_{2}\right) \mid z=x=0\right\} \\
\mathcal{E}_{\left(\mathbb{D}_{2}\right)} & =\left\{\left(z, x, t_{1}, t_{2}\right) \mid z=0, x \neq 0\right\} \\
\mathcal{E}_{\left(\mathbb{Z}_{2}\left(\kappa_{1}\right)\right)}=\left\{\left(z, x, t_{1}, t_{2}\right) \mid z= \pm \bar{z}, z \neq 0\right\}, & \mathcal{E}_{\left(\mathbb{Z}_{2}\left(\kappa_{2}\right)\right)}=\left\{\left(z, x, t_{1}, t_{2}\right) \mid z= \pm i \bar{z}, z \neq 0, x=t_{1}=0\right\}, \\
\mathcal{E}_{e} & =\left\{\left(z, x, t_{1}, t_{2}\right) \mid t_{1}+t_{2} x\left(z^{2}+\bar{z}^{2}\right)=0, z \neq 0, z \neq \pm \bar{z} \text { and, } z \neq \pm i \bar{z} \text { or } x \neq 0\right\} .
\end{aligned}
$$

The manifold $\mathcal{E}_{e}$ has four strata

$$
\begin{aligned}
& S_{e}^{3}=\left\{\left(z, x, t_{1}, t_{2}\right) \in \mathcal{E}_{e} \mid t_{1}=t_{2}=0\right\}, \quad S_{e}^{\prime 3}=\left\{\left(z, x, t_{1}, t_{2}\right) \in \mathcal{E}_{e} \mid t_{1}=0, t_{2} \neq 0, x=0\right\} \\
& S_{e}^{\prime \prime 3}=\left\{\left(z, x, t_{1}, t_{2}\right) \in \mathcal{E}_{e} \mid t_{1}=0, t_{2} \neq 0, x \neq 0, z \neq 0, z= \pm i \bar{z}\right\} \\
& S_{e}^{5}=\left\{\left(z, x, t_{1}, t_{2}\right) \in \mathcal{E}_{e} \mid t_{1} \neq 0, t_{2} \neq 0, x=\frac{-t_{1}}{t_{2}\left(z^{2}+\bar{z}^{2}\right)}\right\} .
\end{aligned}
$$

The strata $S_{e}^{3}, S_{e}^{\prime 3}$ and $S_{e}^{5}$ cover $W_{1}$ while $S_{e}^{\prime \prime 3}$ does not cover $W_{1}$ again due to the fact that $z= \pm i \bar{z}$. Note that $S_{e}^{\prime 3}$ is again the only stratum which defines a manifold as in (14). The stratification of $\mathcal{E}_{G}$ is given by

$$
\begin{aligned}
& S_{G}^{0}=\overline{S_{e}^{3}} \cap \mathbb{R}^{2}=\left\{\left(0,0, t_{1}, t_{2}\right) \mid t_{1}=t_{2}=0\right\}, \quad S_{G}^{1}=\left\{\left(0,0, t_{1}, t_{2}\right) \mid t_{2}=0, t_{1} \neq 0\right\} \\
& S_{G}^{\prime 1}=\overline{S_{e}^{\prime 3}} \cap \mathbb{R}^{2}=\left\{\left(0,0, t_{1}, t_{2}\right) \mid t_{1}=0, t_{2} \neq 0\right\}, \quad S_{G}^{2}=\left\{\left(0,0, t_{1}, t_{2}\right) \mid t_{1} \neq 0, t_{2} \neq 0\right\} .
\end{aligned}
$$

Note that $\overline{S_{e}^{5}} \cap \mathbb{R}^{2}=\overline{S_{e}^{\prime 3}} \cap \mathbb{R}^{2}=S_{G}^{\prime 1}$, so $S_{G}^{0}$ and $S_{G}^{\prime 1}$ are the only central strata.

We can obtain the manifold $K$ at an organizing centre in two ways depending on which central strata graph $f_{2}(0,0)$ intersects in $\mathcal{E}_{\mathbb{D}_{4}}$. As above, if graph $f_{2}(0,0)$ intersects $S_{G}^{0}$, this corresponds to the situation described in Theorem 6.4 and the mapping defining $K$ is

$$
P(z, x, \lambda)=(p(x, z, \lambda), q(x, z, \lambda)) .
$$

The transversality condition for Theorem 6.4 is the same as in the previous example.

If graph $f_{f_{2}}(0,0)$ intersects $S_{G}^{\prime 1}$, this corresponds to the context of Theorem 6.10 and the mapping defining $K$ is

$$
\mathcal{P}(z, \lambda)=p(0, z, \lambda) \text {. }
$$


The transversality condition is

$$
\left(d_{\lambda} \mathcal{P}\right)_{(0,0)}=\frac{\partial p}{\partial \lambda}(0,0,0) \neq 0
$$

and from the implicit function theorem, there exists a smooth $G$-invariant mapping $\psi: \mathbb{C} \rightarrow \mathbb{R}$ with $\psi(0)=0$ such that $\mathcal{P}(z, \psi(z)) \equiv 0$. Thus, $K$ is a two-dimensional manifold defined near $(z, x, \lambda)=(0,0,0)$ by $\{(z, x, \lambda) \in V \times \mathbb{R} \mid x=0, \lambda=\psi(z)\}$.

Concerning $S_{e}^{5}$, one can verify that $\overline{S_{e}^{5}}$ does not form a manifold near $(z, x, t)=(0,0,0)$ and so $\operatorname{graph}_{f_{2}}^{-1}\left(\overline{S_{e}^{5}}\right)$ is generically not a manifold. Thus, we do not obtain an organizing centre in this case, even though $S_{e}^{5}$ covers $W_{1}$.

\section{Acknowledgements}

The authors would like to thank Mike Field for his invaluable advice, suggestions and comments, in particular regarding $G$-transversality theory. We would also like to thank the referees for their suggestions. This research has been supported by the UK Engineering and Physical Sciences Research Council (EPSRC) (PLB,JSWL), the Nuffield Foundation (JSWL), NSERC Postdoctoral Fellowship and NSERC Discovery Grant (PLB), and NSERC Discovery Grants from J. Bélair and C. Rousseau (PLB). PLB and JSWL gratefully acknowledge the hospitality and support of the Department of Mathematics, Imperial College London, the Mathematics Institute, University of Warwick, and the CRM, Montréal, during visits at which part of the research was carried out.

\section{References}

[1] E. Bierstone. General Position of Equivariant Maps. Trans. AMS 234(2) (1977) 447-466.

[2] S. Bochner. Compact groups of differentiable transformations. Ann. of Math. (2) 46 (1945), $372-381$.

[3] C.A. Buzzi and J.S.W. Lamb. Reversible equivariant Hopf bifurcation. Arch. Rat. Mech. Anal. 175 (2005), 39-84.

[4] M.C. Ciocci. Bifurcation of periodic orbits and persistence of quasi periodic orbits in families of reversible systems. PhD Thesis, University of Gent. 2003.

[5] J.C. Da Rocha Medrado and M.A. Teixeira. Symmetric singularities of reversible vector fields in dimension three. Physica D 112 (1998), 122-131.

[6] M.J. Field. Transversality in G-manifolds. Trans. AMS 231(2) (1977), 429-450.

[7] M.J. Field. Stratifications of equivariant varieties. Bull. Aust. Math. Soc. 16 (1977), 279-295.

[8] M.J. Field. Equivariant Bifurcation Theory and Symmetry Breaking. J. Dyn. Diff. Eq, 1, (1989), 369-421.

[9] M.J. Field. Lectures on bifurcations, dynamics and symmetry. Pitman Research Notes in Mathematics Series, 356, Longman, Harlow, 1996. 
[10] M.J. Field. Symmetry-Breaking for Compact Lie Groups. Memoirs of the AMS. 574 (1996).

[11] M.J. Field. Dynamics and Symmetry. Imperial College Press, to appear 2007.

[12] M.J. Field and R.W. Richardson. Symmetry-Breaking and the Maximal Isotropy Subgroup Conjecture for Reflection Groups. Arch. Rational Mech. Anal. 105 (1989), 61-94.

[13] M.J. Field and R.W. Richardson. Symmetry-Breaking and Branching Patterns in Equivariant Bifurcation Theory I. Arch. Rational Mech. Anal. , (1989), 297-348.

[14] M.J. Field and R.W. Richardson. Symmetry-Breaking and Branching Patterns in Equivariant Bifurcation Theory II. Arch. Rational Mech. Anal. 120, (1992), 147-190.

[15] J.-E. Furter, A.M. Sitta and I. Stewart. Singularity theory and equivariant bifurcation problems with parameter symmetry. Math. Proc. Camb. Phil. Soc. 120 (1996), 547-578

[16] C. Gibson, K. Wirthmüller, A. du Plessis, E. Looijenga. Topological stability of smooth mappings. Lecture Notes in Mathematics, 552, Springer-Verlag, Berlin-New York, 1976.

[17] M. Golubitsky, M. Krupa and C. Lim. Time-reversibility and particle sedimentation. SIAM J. App. Math. 51 (1990), 49-72.

[18] M. Golubitsky and D.G. Schaeffer. Singularities and Groups in Bifurcation Theory: Vol.I. Appl. Math. Sci. 51, Springer-Verlag, New York, 1985.

[19] M. Golubitsky, I. Stewart and D.G. Schaeffer. Singularities and Groups in Bifurcation Theory: Vol.II. Appl. Math. Sci. 69, Springer-Verlag, New York, 1988.

[20] V. Guillemin and A. Pollack. Differential Topology Prentice-Hall, Inc. Englewood Cliffs, NewJersey, 1974.

[21] J. Hermans. A symmetric sphere rolling on a surface. Nonlinearity 8 (1995), 493-515.

[22] J.S.W. Lamb and H.W. Capel. Local bifurcations on the plane with reversing point group symmetry. Chaos, Solitons and Fractals 5 (1995), 271-293.

[23] J.S.W. Lamb and I. Melbourne. Bifurcation from discrete rotating waves. Arch. Rat. Mech. Anal. 149 (1999), 229-270.

[24] J.S.W. Lamb, I. Melbourne and C. Wulff. Bifurcation from periodic solutions with spatiotemporal symmetry, including resonances and mode-interactions. J. Differential Equations 191 (2003), 377-407.

[25] J.S.W. Lamb and J.A.G. Roberts. Time-reversal symmetry in dynamical systems: a survey. Physica D 112 (1998), 1-39.

[26] J.S.W. Lamb and M. Roberts. Reversible equivariant linear systems. J. Differential Equations 159, (1999) 239-279. 
[27] J.S.W. Lamb and C. Wulff. Reversible relative periodic orbits. J. Differential Equations 178 (2002), 60-100.

[28] C.C. Lim and I-H. McComb. Time-reversible and equivariant pitchfork bifurcation. Physica D 112 (1998), 117-121.

[29] C.C. Lim and I-H. McComb. Two typical steady-state bifurcations for time-reversible vector field families. J. Dynam. Differential Equations 13 (2001), 251-274.

[30] I.H. McComb and C. Lim. Stability of equilibria for a class of time-reversible, $\mathbb{D}_{n} \times \mathbf{O}(2)$ symmetric homogeneous vector fields. SIAM J. Math. Anal. 24 (4) (1993), 1009-1029.

[31] V. Poénaru. Singularités $C^{\infty}$ en présence de symétrie. Lecture Notes in Mathematics, 510 Springer-Verlag, Berlin-New York, 1976.

[32] A. Politi, G.L. Oppo and R. Badii. Coexistence of conservative and dissipative behaviour in reversible dynamical systems. Phys. Rev. A 33 (1986), 4055-4060.

[33] J.A.G. Roberts and J.S.W. Lamb. Self-similarity of period-doubling branching in 3-D reversible dynamical systems. Physica D 82 (1995), 317-332.

[34] G. Schwarz. Smooth functions invariant under the action of a compact Lie group. Topology, 14, (1975), 63-68.

[35] M.A. Teixeira. Singularities of reversible vector fields. Physica D 100 (1997), 101-118.

[36] A. Vanderbauwhede. Local bifurcation and symmetry. Res. Notes Math. 75, Pitman, Boston, 1982.

[37] A. Vanderbauwhede. Bifurcation of subharmonic solutions in time-reversible systems. $Z$. Angew. Math. Phys. 37 (1986), 455-478.

[38] A. Vanderbauwhede. Hopf bifurcation for equivariant conservative and time-reversible systems. Proc. Roy. Soc. Edinburgh A116 (1990), 103-128.

[39] A. Vanderbauwhede. Subharmonic branching in reversible systems. SIAM J. Math. Anal. 21 (1990), 954-979.

[40] P.A. Worfolk. Zeros of equivariant vector fields: Algorithms for an invariant approach. J. Symb. Comp. 17, (1994), 487-511.

[41] C. Wulff, J.S.W Lamb and I. Melbourne. Bifurcation from relative periodic solutions. Ergodic Theory Dynam. Systems 21 (2001), 605-635. 